\title{
Didactic application of numerical analysis in nonlinear dynamics: Lorenz model study
}

\section{Aplicación didáctica del análisis numérico en dinámica no lineal: estudio del modelo de Lorenz}

\author{
F.V. Garcia-Ferrer ${ }^{1 *}$, E. Roldán ${ }^{1}$, F. Silva ${ }^{1, S}$ and G.J. de Valcárcel1,S. \\ 1. Departament d' Optica, Universitat de València, Dr. Moliner 50, 46100-Burjassot, Spain \\ ${ }^{(*}$ E-mail: ferran.garcia@uv.es. \\ S: SEDOPTICA member \\ Received: 01/08/2016 Accepted: 06/04/2017 \\ DOI: 10.7149/OPA.50.3.49009
}

\begin{abstract}
:
We describe a practice designed for the numerical study of the Lorenz model that is a central model in the physics of lasers. The didactical objectives pursued in this practice have a dual nature, considering both the introduction to the knowledge of a physical paradigm of deterministic chaos as the training for the use of certain computational tools for its characterization. The method used to achieve programming is finding solutions of the Lorenz model and systematically studying of their temporal evolution using a Mathematica program. In the academic context, the practice is designed to be included in the curriculum of the degree in physics and to facilitate adaptation to other matters in this area, such as quantum optics, fluids, mechanical vibrations, etc. We first study the steady states, and their linear stability, of the Lorenz model equations and then numerically study the different types of dynamic behavior. We pay special attention to the deterministic chaotic behavior and to the sequence of bifurcations leading from periodic to chaotic behavior (routes to chaos).
\end{abstract}

Key words: Lorenz model, chaos, nonlinear dynamics, laser, didactic, Mathematica.

\section{RESUMEN:}

Describimos una práctica diseñada para el estudio numérico de un modelo central en la física del láser, el modelo de Lorenz. Los objetivos didácticos que se persiguen en la práctica tienen una naturaleza doble, por un parte, la introducción al conocimiento de un paradigma físico del caos determinista y, por otra, la capacitación para la utilización de ciertas herramientas computacionales que permiten su caracterización. El método utilizado para alcanzarlos es la búsqueda de las soluciones del modelo de Lorenz y el estudio sistemático de su evolución temporal mediante el programa Mathematica. En el contexto académico, la práctica está diseñada para ser incluida en el currículum del grado en Física y para facilitar su adaptación a otras materias dentro de este ámbito, como puede ser óptica cuántica, fluidos, oscilaciones mecánicas, etc. Partiendo de las ecuaciones del modelo de Lorenz, estudiamos sus estados estacionarios y su estabilidad lineal, para después estudiar numéricamente los distintos tipos de comportamiento dinámico. Prestamos especial atención al comportamiento caótico determinista y a la secuencia de bifurcaciones que lleva de los comportamientos periódicos a los caóticos (rutas al caos).

Palabras clave: Modelo de Lorenz, caos, dinámica no lineal, láser, didáctica, Mathematica.

\section{REFERENCES AND LINKS / REFERENCIAS Y ENLACES}

[1] E.N. Lorenz, "Deterministic nonperiodic flow", J. Atm. Sci. 20, 130-141 (1963). https://doi.org/10.1175/1520-0469(1963)020<0130:DNF>2.0.C0;2

[2] C. Sparrow, The Lorenz equations: Bifurcations, Chaos and Strange Attractors. Berlin-Heidelberg-New York, Springer-Verlag (1982).

https://doi.org/10.1007/978-1-4612-5767-7 
[3] H. Haken, "Analogy between higher instabilities in fluids and lasers", Phys. Lett. A 53, 77-88 (1975).

[4] G.J. de Valcárcel, E. Roldán, y F. Prati, "Semiclassical theory of amplification and lasing", Rev. Mex. Fis. 52, 198-214 (2006).

[5] C.O. Weiss y R. Vilaseca, Dynamics of lasers. Weinheim, VCH (1991).

[6] http://numericalmethods.eng.usf.edu (July, 2016).

[7] G.J. de Valcárcel, E. Roldán y R. Vilaseca "Stability properties of a resonant cascade laser" Phys. Rev. A 49, 1243-1259 (1994). https://doi.org/10.1103/PhysRevA.49.1243

[8] P. Bergé, Y. Pomeau, y C. Vidal, Order Within Chaos: Towards A Deterministic Approach to Turbulence. Paris, John Wiley \& Sons Inc. (1984).

[9] R.L. Devaney, An Introduction to Chaotic Dynamical Systems. Redwood City, Addison-Wesley (1989).

[10] K. Falconer, Fractal Geometry: Mathematical Foundations and Applications. Chichester-New YorkBrisbane-Totonto-Singapore, John Wiley \& Sons Inc. (2003). https://doi.org/10.1002/0470013850

[11] C.Z. Ning y H. Haken, "Multistabilities and anomalous switching in the Lorenz-Haken model", Phys. Rev. A 41, 6577-6580 (1990). https://doi.org/10.1103/PhysRevA.41.6577

[12] E. Knobloch, "Chaos in the segmented disc dynamo", Phys. Lett. A 82, 439-440 (1981). https://doi.org/10.1016/0375-9601(81)90274-7

[13] A. Tonjen, R. J. Thelwell y D. Becerra-Alonso, "Reinventing the wheel: the chaotic sandwheel", Am. J. Phys. 81, 127-133 (2013). https://doi.org/10.1119/1.4768893

[14] Yu. I. Neimark y P.S. Landa, Stochastic and chaotic oscillations. Kluwer Academic Publishers (1992). https://doi.org/10.1007/978-94-011-2596-3

[15] P.S. Landa, Regular and chaotic oscillations. Berlin-Heidelberg-New York, Springer-Verlag (2001). https://doi.org/10.1007/978-3-540-45252-2

[16] L. E. Matson, “The Malkus-Lorenz water wheel revisited”, Am. J. Phys. 75, 1114-1122 (2007). https://doi.org/10.1119/1.2785209

[17] W. V. R. Malkus, "Non-periodic convection at high and low Prandtl number", Mem. Soc. R. Sci. Liege Collect IV, 125-128 (1972).

[18] M. Kolar and G. Gumbs, "Theory for the experimental observation of chaos in a rotating waterwheel", Phys. Rev. A 45 626-637 (1992). https://doi.org/10.1103/PhysRevA.45.626

[19] G. Haag, T. Hagel y T. Sigg, "Active Stabilization of a Chaotic Urban System", Discrete Dynamics in Nature and Society 1, 127-134 (1997). https://doi.org/10.1155/S1026022697000137

[20] G. Gabish y H.W. Lorenz, Business Cycle Theory. New York, Springer (1987). https://doi.org/10.1007/978-3-662-01178-2

[21] H.W. Lorenz, Nonlinear dynamical economics and chaotic motion. Berlin-Heidelberg, Springer-Verlag (1989). https://doi.org/10.1007/978-3-662-22233-1

[22] C.O. Weiss y J. Brock, "Evidence for Lorenz-Type Chaos in a Laser", Phys. Rev. Lett. 57, 2804-2806 (1986). https://doi.org/10.1103/PhysRevLett.57.2804

[23] D. Y. Tang, C. O. Weiss, E. Roldan, and G. J. de Valcarcel, "Deviation from Lorenz-type dynamics of an NH3ring laser," Opt. Commun. 89, 47-53 (1992) https://doi.org/10.1016/0030-4018(92)90246-N

[24] L. Illing, R. F. Fordyce, A. M. Saunders y R. Ormond, "Experiments with a Malkus-Lorenz water wheel: Chaos and Synchronization", Am. J. Phys. 80, 192-202 (2012). https://doi.org/10.1119/1.3680533 


\section{Introducción}

Este trabajo propone unos contenidos y una metodología aplicables en el grado en física con la finalidad de incorporar en su currículum la dinámica no lineal. Esta incorporación se justifica por la enorme importancia en muchas áreas de la ciencia y la tecnología de las ecuaciones diferenciales no lineales (tanto ordinarias como en derivadas parciales); argumento válido también, desde nuestro punto de vista, para los currícula de los grados en química, biología, ciencias o los de las diversas ingenierías. Creemos que, dentro del marco de los planes de estudio actuales, debería incluirse el objetivo de conocer, por lo menos en cierta medida, la riqueza y variedad de las diversas soluciones que albergan las ecuaciones diferenciales no lineales (soluciones estacionarias, dinámicas, caóticas, biestables, etc.). Por otra parte, el caos determinista, que exhiben muchos sistemas no lineales con al menos tres grados de libertad, es también un fenómeno de gran importancia para una adecuada comprensión del alcance del determinismo y de los límites de la predictibilidad y, por tanto, del conocimiento de la ciencia en general y de la física en particular.

Uno de los atractivos del estudio de la dinámica no lineal es su universalidad, esto es, que los mismos comportamientos se presenten en sistemas sorprendentemente diversos (físicos, químicos, biológicos, económicos, etc.), lo que hace que la dinámica no lineal sea altamente multidisciplinar.

Esta universalidad también abarca el estudio de los mecanismos que llevan a la desestabilización de una determinada solución al variar un parámetro en el sistema. Nos referimos a la transición de una a otra solución del sistema dinámico y destacamos que este conjunto, finito y bien delimitado, de mecanismos están descritos con unas matemáticas independientes de la naturaleza del sistema. Estas técnicas matemáticas son el corazón de la dinámica no lineal.

Precisamente, esta universalidad resulta una ventaja a la hora de introducir conceptos de dinámica no lineal en el currículum de física, o en el de cualquier otra disciplina científica: no es necesario plantear el estudio formal de la matemática no lineal como una disciplina aparte, sino que puede introducirse a partir del estudio de casos particulares. De esta forma se adquieren algunas herramientas matemáticas sencillas de gran potencia, tanto analíticas como numéricas, así como nuevas herramientas conceptuales.

Los sistemas dinámicos no lineales se dividen en dos grandes grupos, conservativos y no conservativos, según sea despreciable, o no, el intercambio de energía con el entorno. Aquí nos vamos a centrar en los sistemas no conservativos a través del estudio del célebre modelo de Lorenz. El modelo de Lorenz $[1,2]$ es un conjunto de tres ecuaciones diferenciales reales ordinarias y no lineales gobernadas por tres parámetros. Son muy importantes por ser paradigmáticas y porque constituyen el primer ejemplo histórico de un sistema de ecuaciones diferenciales que presentan el fenómeno del caos determinista, esto es, la imposibilidad de predecir el comportamiento a largo plazo de una variable.

Cuando definimos el comportamiento de un sistema como caos determinista es porque las soluciones de las ecuaciones que describen su evolución muestran sensibilidad a las condiciones iniciales. Esta sensibilidad se observa gráficamente si representamos en el espacio fásico (aquel cuyos ejes ortogonales son las variables del sistema) dos trayectorias o evoluciones de las soluciones que difieren infinitesimalmente en las condiciones iniciales y constatamos que sus trayectorias se alejan exponencialmente entre sí. Esto implica la imposibilidad de predecir exactamente el valor de la variable al cabo de un cierto tiempo, pues las condiciones iniciales siempre tienen una cierta incertidumbre y por pequeña que sea se transformará en una enorme incertidumbre al cabo de un cierto lapso de tiempo característico. Además de ser el primer sistema de ecuaciones diferenciales que presentan caos determinista, el modelo de Lorenz ha sido muy bien estudiado y ha resultado ser aplicable a bastantes sistemas físicos muy diferentes entre si. Originalmente Lorenz lo propuso en 1963 como un modelo atmosférico. Más tarde, en 1975, Haken [3] descubrió que también describe cierto tipo de láseres. Desde entonces son diversos los sistemas que se ha descubierto que vienen descritos por ecuaciones isomorfas a las del modelo de Lorenz, de forma que el modelo ha llegado a asumir el estatus de paradigmático.

En el grado en Física en la Universitat de València, llevamos años incluyendo, en el marco de la asignatura optativa "Óptica Cuántica", diversos contenidos, tanto teóricos como prácticos - en simulación por ordenador -, sobre el modelo de Lorenz. Un curso de óptica cuántica es claramente apropiado por la conexión del modelo de Lorenz con la física del láser a través del modelo de Lorenz-Haken, pero queremos hacer notar que el marco de un curso sobre dinámica de fluidos o de física de la atmósfera o de un curso de mecánica al estudiar oscilaciones mecánicas o de electricidad en el caso de las oscilaciones en 
circuitos caóticos, pueden ser igualmente apropiados, como también lo sería, obviamente, un curso sobre ecuaciones diferenciales.

En nuestro planteamiento didáctico más global, el modelo de Lorenz para un láser [4] es rigurosamente deducido antes de ser estudiado, cosa que no haremos aquí. En este trabajo, dirigido a las sesiones prácticas, prestaremos, en cambio, especial atención a las soluciones del modelo, dado su carácter altamente universal como ya hemos comentado.

De acuerdo con estos principios conceptuales y metodológicos, los objetivos didácticos específicos perseguidos con la práctica son:

- Conocimiento del modelo de Lorenz como paradigma de estudio de la dinámica no lineal.

- Introducción a los conceptos físicos y matemáticos relacionados con los sistemas dinámicos no lineales: espacio fásico, análisis de estabilidad lineal, bifurcaciones, bifurcación de Hopf, evolución adiabática, excitación mediante perturbaciones grandes (hard-mode excitation).

- Introducción y reconocimiento de los conceptos básicos relacionados con el estudio del caos determinista: atractores, rutas al caos y sensibilidad a las condiciones iniciales.

- Conocimiento del programa Mathematica, a nivel de usuario, para la caracterización de soluciones de sistemas de ecuaciones diferenciales no lineales.

Queremos señalar que nuestro enfoque está claramente en deuda con el del texto de Weiss y Vilaseca [5] en el que se aborda una introducción similar, aunque menos extensa y detallada, a la dinámica del modelo de Lorenz.

\section{El modelo de Lorenz}

Propuesto por Edward N. Lorenz en 1963 [1] como una versión muy simplificada de un modelo atmosférico -el de un fluido sometido a un gradiente externo de temperaturas- consiste en el siguiente conjunto de tres ecuaciones diferenciales reales de primer orden, acopladas y no lineales:

$$
\begin{gathered}
\frac{d}{d t} x=\sigma(y-x), \\
\frac{d}{d t} y=-y+x(r-z), \\
\frac{d}{d t} z=-b z+x y,
\end{gathered}
$$

Las tres variables adimensionales $x, y, z$, son proporcionales a diferentes magnitudes físicas según sea el sistema en que se implementa el modelo de Lorenz, mientras que $\sigma, b, r$ son parámetros reales y los dos primeros positivos (o nulos en el límite), puesto que son tasas de relajación.

Cabe subrayar, además, como se deduce de la coherencia dimensional entre los términos de las ecuaciones, que la variable $t$ está normalizada con la tasa de relajación de la variable $y$, de forma que el tiempo es adimensional como también lo son $\sigma, b$; las tasas de relajación de $x$, $z$. En el caso del modelo de dinámica de fluidos originalmente ideado por Lorenz, $x$ e $y$ son la primera y segunda componentes de Fourier del campo de velocidades; $z$ es la primera componente de Fourier del campo de temperatura del fluido. En el caso de la física del láser (modelo de Lorenz-Haken [3]) $x$ representa la amplitud del campo eléctrico de la radiación luminosa, de forma que $x^{2}$ es proporcional, por tanto, a la irradiancia del haz láser; mientras que $y, z$ representan propiedades de los átomos del medio activo en el que ocurre la amplificación láser; en concreto, la variable y es proporcional al dipolo inducido en los átomos por la radiación luminosa, y la $z$ es proporcional a la inversión de población cambiada de signo (se pueden ver más detalles en [4]). En cuanto a los parámetros, $b$ es el cociente entre la tasa de relajación de la inversión de población y la del dipolo inducido, lo que hace que en el contexto óptico $b \leq 1$; mientras que $\sigma$ es el cociente entre la tasa de relajación del campo y la del dipolo y su valor no está limitado. El parámetro de bombeo $r$ es el cociente entre las ganancias y las pérdidas que tiene el proceso de amplificación estimulada, definido de forma tal que $r=1$ (igualdad entre ganancia y pérdidas) corresponde con el umbral de emisión láser, como se verá más abajo. 
Es interesante señalar una simetría presente en el modelo: las ecuaciones (1) son invariantes al cambio $(\mathrm{x}, \mathrm{y}, \mathrm{z}) \rightarrow(-\mathrm{x},-\mathrm{y}, \mathrm{z})$.

\section{2.a Soluciones estacionarias}

Las ecuaciones del modelo de Lorenz no pueden resolverse analíticamente en su forma general. En un caso particular, no obstante, podemos conocerlas con toda generalidad; es el caso de las soluciones independientes del tiempo, o estacionarias, que se obtienen sin más que anular las derivadas en las ecuaciones (1a-1c). Obtenemos así la solución trivial:

$$
\bar{x}=\bar{y}=\bar{z}=0
$$

y las soluciones no triviales:

$$
\bar{x}=\bar{y}= \pm \sqrt{b(r-1)}, \bar{z}=r-1,
$$

donde la barra sobre los símbolos denota que se trata de soluciones estacionarias.

En términos de física del láser diríamos que la solución trivial se corresponde con la situación de láser apagado, mientras que las soluciones no triviales se corresponden con la solución de láser encendido y emisión $c w$ (continuous wave, con intensidad constante). Es interesante destacar que la solución trivial existe para cualquier valor de los parámetros, mientras que las no triviales requieren un valor mínimo en la tasa de bombeo: $r>1$; lo que desde un punto de vista físico implica que para encender el láser debemos superar un determinado umbral en el valor del bombeo (concretamente, las ganancias han de superar a las pérdidas).

Observamos también que para $r>1$ existen simultáneamente las dos soluciones estacionarias. Dada esta coexistencia, cabe preguntarse ¿qué solución elige el sistema y qué decide la elección? Por otra parte, aparte de las soluciones estacionarias, deben existir soluciones de las ecuaciones de Lorenz también en el caso de no eliminar la dependencia del tiempo, ¿podemos decir algo acerca de esas soluciones?

Para contestar ambas preguntas es necesario utilizar el análisis lineal de estabilidad.

\section{2.b Análisis de estabilidad lineal de las soluciones estacionarias. Bifurcaciones}

El análisis lineal de estabilidad es una técnica matemática que informa con precisión de en qué lugares del espacio de parámetros las distintas soluciones analizadas son estables o inestables frente a pequeñas perturbaciones.

La idea se puede exponer de forma intuitiva si consideramos la analogía entre las soluciones estacionarias y los puntos de equilibrio de un potencial: los lugares en los que éste alcanza un máximo o un mínimo son puntos de equilibrio, pero en los máximos el equilibrio es inestable y cualquier pequeña perturbación se amplifica exponencialmente, mientras que en los mínimos no ocurre así. Siguiendo la analogía, lo que ocurre en las bifurcaciones es que una solución estacionaria pasa de ser el análogo de un mínimo de potencial (pues atrae hacia sí las perturbaciones y por eso recibe el nombre de atractor) a ser el análogo de un máximo de potencial (de forma que ya no es un atractor).

El análisis lineal de estabilidad, por tanto, permite ver cómo evolucionan pequeñas perturbaciones de una solución, por ejemplo, la estacionaria. Para realizar este análisis procedemos a una sustitución en las ecuaciones $(1 \mathrm{a}-1 \mathrm{c})$ :

$$
\begin{aligned}
& x(t)=\bar{x}+\delta x(t), \\
& y(t)=\bar{y}+\delta y(t), \\
& z(t)=\bar{z}+\delta z(t)
\end{aligned}
$$

entendiendo que $\delta x(t)$, etc. son cantidades pequeñas, con lo que pueden despreciarse cantidades del orden de $\delta x(t)^{2}$ o menores. El resultado de esa operación deja las siguientes ecuaciones de Lorenz linealizadas para las perturbaciones

$$
\frac{d}{d t} \delta x=\sigma(\delta y-\delta x)
$$




$$
\begin{gathered}
\frac{d}{d t} \delta y=-\delta y+(r-\bar{z}) \delta x-\bar{x} \delta z, \\
\frac{d}{d t} \delta z=-b \delta z+\bar{y} \delta x-\bar{x} \delta y
\end{gathered}
$$

O abreviadamente

$$
\frac{d}{d t} \overrightarrow{\delta x}=\hat{L} \overrightarrow{\delta x}
$$

con $\delta \vec{x}=\operatorname{col}(\delta x, \delta y, \delta z)$ y la matriz

$$
\hat{L}=\left(\begin{array}{ccc}
-\sigma & \sigma & 0 \\
r-\bar{z} & -1 & -\bar{x} \\
\bar{y} & \bar{x} & -b
\end{array}\right) .
$$

Las soluciones propias de las ecuaciones $(6,7)$ son de la forma

$$
\overrightarrow{\delta x}(t)=\sum_{i=1}^{3} \overrightarrow{\delta x_{i}}(0) \cdot e^{\lambda_{i} t}
$$

donde los $\overrightarrow{\delta x_{i}}(0)$ son proporcionales a los autovectores de la matriz $\hat{L}$ y los $\lambda_{i}$ son sus correspondientes autovalores, que dependen de los parámetros del sistema $r, \sigma, b$ y de la solución estacionaria analizada $(\bar{x}, \bar{y}, \bar{z})$. Es claro que es la parte real de los autovalores la que determina la estabilidad de la solución considerada: si $\operatorname{Re}\left(\lambda_{i}\right)<0$ para $i=1,2,3$, la solución es estable, pues todas las perturbaciones se amortiguan exponencialmente con el tiempo. Pero si $\operatorname{Re}\left(\lambda_{i}\right)>0$ para algún $i$, la solución deviene inestable, ya que las perturbaciones crecerán exponencialmente a lo largo de la dirección del autovector correspondiente en el espacio fásico. Se denomina bifurcación al lugar, en el espacio de parámetros, en el que $\operatorname{Re}\left(\lambda_{i}\right)=0$.

Existen diversos tipos de bifurcaciones, pero aquí nos vamos a encontrar únicamente con dos: una, denominada de horca (pitchfork en inglés), en la que $\operatorname{Re}\left(\lambda_{i}\right)=\operatorname{Im}\left(\lambda_{i}\right)=0$, y otra, llamada de Hopf, en la que $\operatorname{Re}\left(\lambda_{i}\right)=0$ pero $\operatorname{Im}\left(\lambda_{i}\right) \neq 0$. La primera es una bifurcación estática en la que no aparecen oscilaciones $\mathrm{y}$ señala el cambio de estabilidad entre dos soluciones estacionarias. La segunda es una bifurcación dinámica pues en ella hay oscilaciones de frecuencia $\Omega_{i}=\operatorname{Im}\left(\lambda_{i}\right)$, tal y como se deduce de la expresión (8) $\operatorname{con} \lambda_{i}=i \Omega_{i}$.

\section{Análisis de estabilidad de la solución trivial}

Sustituyendo en la matriz de estabilidad (7) la solución trivial (3) se obtiene una matriz cuyos autovalores son:

$$
\begin{gathered}
\lambda_{0}=-b, \\
\lambda_{ \pm}=-(\sigma+1) / 2 \pm \sqrt{(\sigma-1)^{2} / 4+\sigma r},
\end{gathered}
$$

El primer autovalor indica que las perturbaciones en la variable $z$ están exponencialmente amortiguadas con una velocidad igual a su tasa de decaimiento. Los otros dos autovalores son reales, pero sólo un autovalor, $\lambda_{+}$, puede ser positivo. Resolviendo $\lambda_{+} \geq 0$ obtenemos que esto ocurre cuando $r \geq 1$.

Así pues, si incrementamos $r$ desde cero, la solución trivial es inicialmente estable, pero cuando $r=1$ ocurre una bifurcación de horca y para $r$ mayor que la unidad la solución trivial es inestable. Dado que se trata de una bifurcación estática, la pérdida de estabilidad debe ir asociada a una transición a otra solución estacionaria, y así es como ocurre ya que en $r=1$ las soluciones no triviales (3) empiezan a existir y para un $r$ superior, pero en las proximidades de $r=1$, estas soluciones no triviales son estables como veremos a continuación. 


\section{Análisis de estabilidad de las soluciones no triviales}

Sustituyendo ahora en la matriz (7) las soluciones no triviales (3) tenemos una nueva matriz cuyos autovalores son los mismos para ambas soluciones y vienen dados por la ecuación característica

$$
\lambda^{3}+(\sigma+b+1) \lambda^{2}+b(r+\sigma) \lambda+2 b \sigma(r-1)=0
$$

La igualdad en los autovalores que hemos señalado implica que la estabilidad de las dos soluciones no triviales es la misma, y, por tanto, hablaremos en singular de la solución no trivial. Esta igualdad es consecuencia de la simetría, ya señalada, del modelo.

En este caso las expresiones explícitas de los autovalores no proporcionan información útil, lo que no impide que podamos obtener la información que nos interesa: conocer, para el caso en que la parte real del autovalor se hace cero (bifurcación), si su parte imaginaria es nula o no.

Esto lo podemos resolver imponiendo que $\lambda$ sea un número imaginario puro: $\lambda=i \operatorname{Im}(\lambda) \equiv i \Omega$. En ese caso, las dos soluciones para $\Omega$ y para $r$ son

$$
\begin{gathered}
r=r_{0} \equiv 1, \quad \Omega \equiv 0 ; \\
r=r_{H B} \equiv \frac{(3+b+\sigma) \sigma}{\sigma-1-b},
\end{gathered}
$$

Vemos que ésta puede sufrir dos bifurcaciones: una bifurcación de horca para $r=1$ (que es la misma que hemos encontrado al analizar la estabilidad de la solución trivial sólo que vista desde el otro lado); y una bifurcación de Hopf para $r=r_{H B}$. Representamos estas bifurcaciones y soluciones en la Fig. 1.

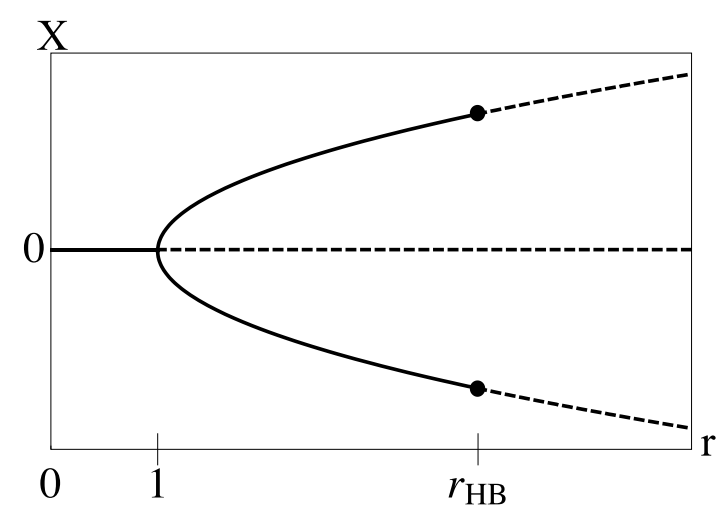

Fig. 1. Representación del valor de $X$ en las soluciones estacionarias de las ecuaciones de Lorenz en función del parámetro de bombeo $r$. Representamos con línea continua las zonas de estabilidad y con discontinua las zonas de inestabilidad.

Mientras que la bifurcación de horca $(r=1)$ existe para cualesquiera valores de los parámetros de relajación $\sigma$ y $b$, la bifurcación de Hopf no existe salvo que $\sigma>\sigma_{m c} \equiv b+1$ (ya que $\Omega^{2}{ }_{H B}$ ha de ser positivo necesariamente), es decir las pérdidas de la variable $x$ han de superar el valor mínimo $\sigma_{m c}$. En el contexto de la física del láser, nos referimos a esta condición como "de mala cavidad", pues, en ese sistema óptico, $\sigma$ representa las pérdidas que sufre el campo electromagnético en el interior de la cavidad láser a través del acoplador o espejo no perfecto del láser (el espejo que deja salir al exterior parte de la radiación).

Así pues, tal y como representamos en la Fig. 2, tenemos un mapa global sobre la estabilidad de las soluciones estacionarias: (i) la solución trivial existe siempre pero sólo es estable para $r<1$; (ii) la solución no trivial existe para $r \geq 1$ y es estable siempre si $\sigma<\sigma_{m c}$ y (iii) si $\sigma>\sigma_{m c}$ la solución no trivial pierde la estabilidad en $r=r_{H B}$ a través de una bifurcación de Hopf. Dado que para $r>r_{H B}$ ninguna solución estacionaria es estable, en ese dominio de bombeos sólo podemos esperar que existan soluciones dinámicas. Tal como hemos señalado, representamos un mapa global sobre la estabilidad de estas soluciones en la Fig. 2.

Hasta aquí la información que podemos obtener analíticamente de forma sencilla. Esta información se puede ampliar con el estudio analítico de la estabilidad de las soluciones periódicas que sabemos que existen en la bifurcación de Hopf [7]. Sin embargo, esto queda fuera del alcance de la práctica que proponemos en el curso y que presentamos en este artículo. 


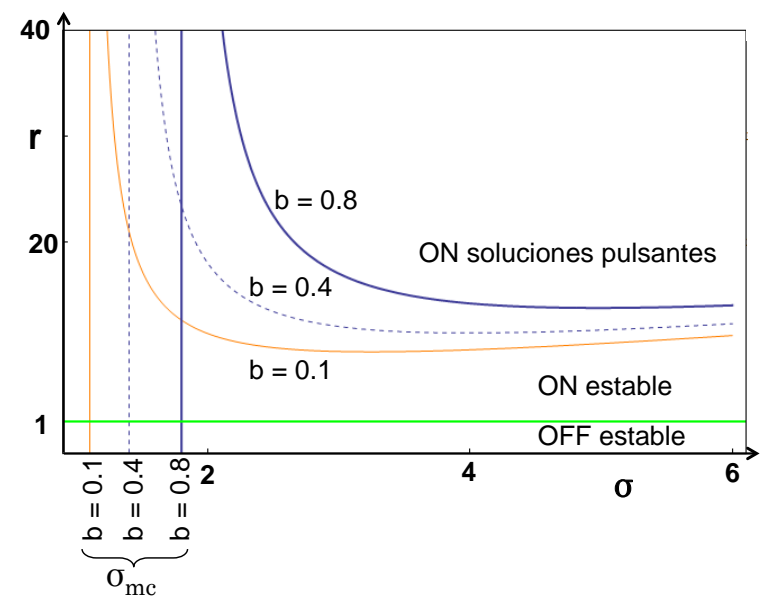

Fig. 2. Valor de la bifurcación de Hopf para diversos valores de $b$ en función de $\sigma$ y de $r$. Para mayor claridad, no se ha respetado la proporcionalidad en la escala para situar $r=1$.

\section{2.c Estudio numérico del modelo de Lorenz}

¿Qué tipo de soluciones existen más allá de la bifurcación de Hopf? ¿Cuándo $r>1$ encontraremos siempre la solución estacionaria no trivial si no hemos cruzado la bifurcación de Hopf [i.e., si $r<r_{H B}$, Ec. (12)], o existen también otras soluciones dinámicas que coexisten con la solución estacionaria? La contestación a estas preguntas requiere la integración numérica de las ecuaciones del modelo, lo que se puede llevar a cabo mediante el método de Runge-Kutta [6], por ejemplo. Sin embargo, en nuestra aproximación didáctica consideramos más práctico utilizar algún programa comercial como Mathematica o Matlab. Hemos optado por Mathematica debido a que hemos comprobado que los alumnos alcanzan, en el desarrollo de la práctica, las competencias necesarias para utilizar su sintaxis y llevar a cabo los cálculos necesarios.

Como los objetivos didácticos que perseguimos no incluyen el estudio ni el conocimiento en profundidad del programa Mathematica, a los estudiantes se les entrega el notebook -archivo propio del programa- con los elementos básicos necesarios para la práctica y, además, para facilitar su uso impartimos una sesión previa en el aula de informática dirigida a familiarizar a los estudiantes con su sintaxis básica.

En este apartado, por tanto, describiremos los casos estudiados, el motivo por el cual se han escogido y cuáles son las conclusiones que se pueden extraer de los resultados obtenidos, sin entrar en el detalle de cómo se programa su desarrollo y solución en el programa de cálculo escogido.

\section{Evolución temporal y estabilidad}

A través de la representación gráfica de la evolución temporal del sistema, podemos observar con más claridad qué significa estabilidad y apuntar qué tipo de respuestas encontramos para las preguntas planteadas al inicio de este apartado. En concreto, estudiamos la evolución temporal de una variable con un solo parámetro libre. Elegimos el caso de la solución no trivial y fijamos, como ejemplo para toda la práctica, el valor de dos de los parámetros $(b=1$ y $\sigma=3)$ de forma que el tercero $(r$, el parámetro de bombeo) será el parámetro de control. Por otra parte, en un experimento real, $r$ podría variarse o bien poco a poco (variación adiabática o soft-mode excitation) o bien bruscamente (hard-mode excitation). Numéricamente, la primera opción -SME- se implementa integrando las ecuaciones a partir de unas condiciones iniciales (c.i.) para las variables próximas al valor estacionario; mientras que la hard-mode excitation -HME- se suele implementar utilizando unas c.i. alejadas del estado estacionario correspondiente.

Para los valores de los parámetros escogidos obtenemos, de acuerdo con (12), $r_{H B}=21$. Por tanto, comenzamos a variar $r$ de forma adiabática desde $r=10$. Distintos comportamientos de la solución no trivial pueden apreciarse en la Fig. 3, donde representamos la evolución temporal de $X^{2}$, proporcional a la intensidad del láser, para el valor inicial de $r$ elegido y también para $r=10 ; r=18 ; r=20,2$ y $r=20,95$.

Se observa que a medida que $r$ aumenta, el régimen transitorio, o tiempo que el sistema tarda en alcanzar la solución estable, es cada vez más largo y para valores superiores a la bifurcación, esta solución ya no se alcanza nunca. 

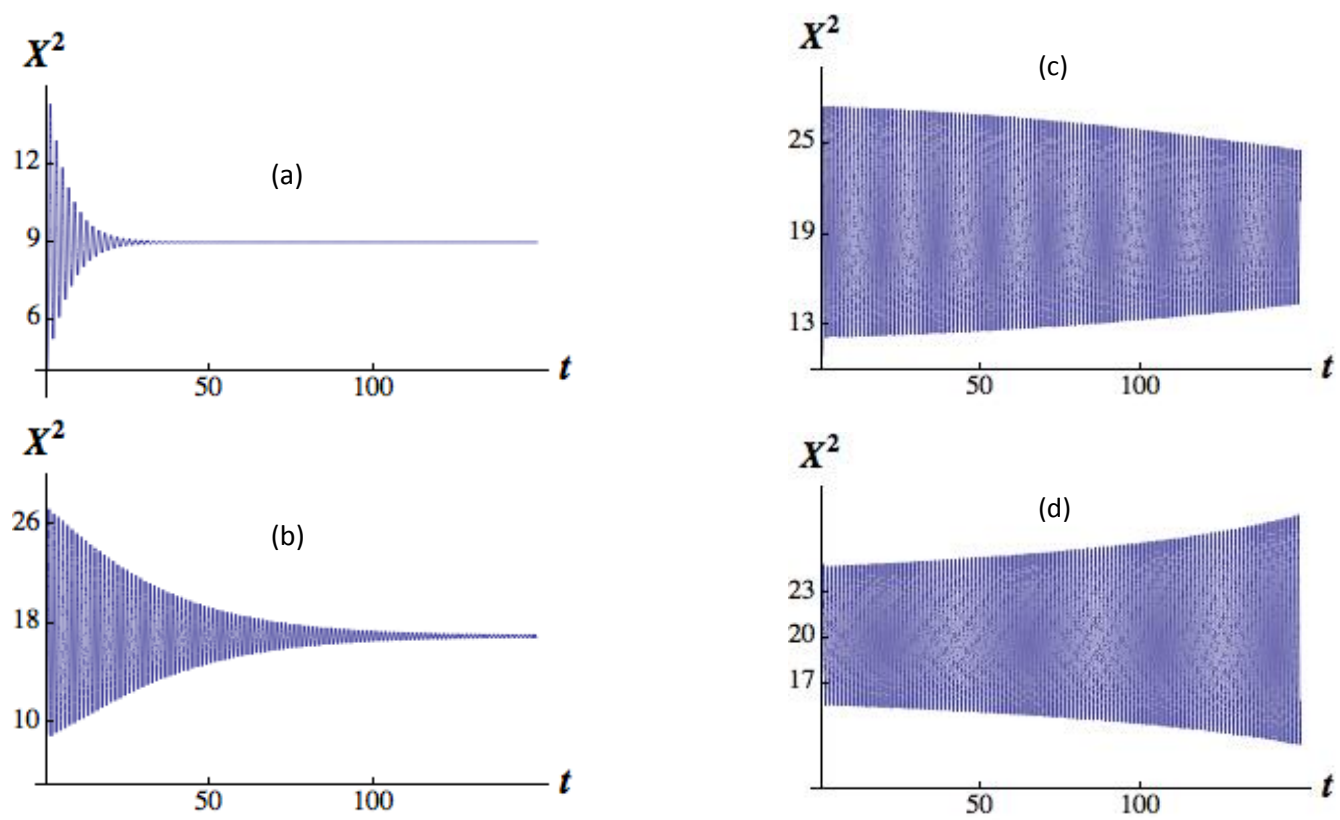

Fig. 3. Evolución temporal de $X^{2}$ para cuatro bombeos $r$ inferiores al de la bifurcación de Hopf $\left[r_{H B}=21\right.$ para $\left.\sigma=3 ; b=1\right]$. (a) SME, con c.i. $\left\{X_{0}=Y_{0}=2 ; Z_{0}=8\right\}$ y $r=10$; se observa que tras un transitorio breve el sistema decae a la solución estacionaria $X^{2}=9$. (b) SME, con c.i. $\left\{X_{0}=Y_{0}=3 ; Z_{0}=15\right\}$ y $r=18$; se observa que tras un transitorio un poco más largo que en el caso anterior el sistema "cae" a la solución estacionaria $X^{2}=16,7$. (c) SME, con c.i. $\left\{X_{0}=Y_{0}=3,3 ; Z_{0}=18,2\right\}$ y $r=20,2$; se observa que el sistema "cae" mucho más lentamente a la solución estacionaria $X^{2}=19,18$. (d) c.i. $\left\{X_{0}=Y_{0}=4,2 ; Z_{0}=18,7\right\}$ y $r=20,95$.

Este resultado es acorde con lo que hemos visto en el análisis de estabilidad lineal, donde se prevé que las perturbaciones evolucionarán como $\exp \left[\operatorname{Re}\left(\lambda_{\max }\right) t\right]$, lo que implica un tiempo de decaimiento de las perturbaciones de $\tau \sim\left(\operatorname{Re}\left(\lambda_{\max }\right)\right)^{-1} \mathrm{y}$, por tanto, cuando $r$ es próxima a $r_{H B}$ el transitorio se vuelve muy largo (ver la Fig. 3(c)). Para $r \approx r_{H B}$ se observa que las oscilaciones ya no son de relajación, es decir, ya no son únicamente un transitorio que acaba en una solución estacionaria. Para estos valores de $r$, próximos a la bifurcación, las mismas perturbaciones que llevaban al sistema a evolucionar hasta la solución estable, ahora pueden conducir a la desestabilización de la misma (ver la Fig. 3(d)). Esto es debido a que cerca de la bifurcación de Hopf (12) la solución estacionaria es muy poco estable, es decir, porque su "cuenca de atracción" (el conjunto de condiciones iniciales que acaban en esa solución) es muy reducida cerca de la bifurcación.

Concluimos, por tanto, que, si las perturbaciones son pequeñas, o lo que es lo mismo, la condición inicial es suficientemente próxima al estado estacionario que corresponde al valor de los parámetros y nos encontramos dentro de la zona de estabilidad, las soluciones obtenidas decaen a las estacionarias obtenidas por el análisis de estabilidad lineal. Sin embargo, sabemos que si sobrepasamos el valor de la bifurcación para el parámetro de control $\left(r_{H B}\right)$, las perturbaciones crecen exponencialmente y al cabo de cierto tiempo el sistema acaba en otra solución dinámica. Veamos ahora cómo es este otro tipo de solución.

\section{Evolución temporal fuera de la zona de estabilidad}

Dado que podemos llegar a este otro tipo de solución partiendo de valores del bombeo tanto inferiores, como superiores a la bifurcación, hemos elegido dos ejemplos correspondientes a cada uno de estos dos caminos.

Vemos en primer lugar qué sucede con un bombeo inferior a la bifurcación, pero con una perturbación suficientemente grande, o equivalentemente una variación de $r$ no suficientemente lenta. 


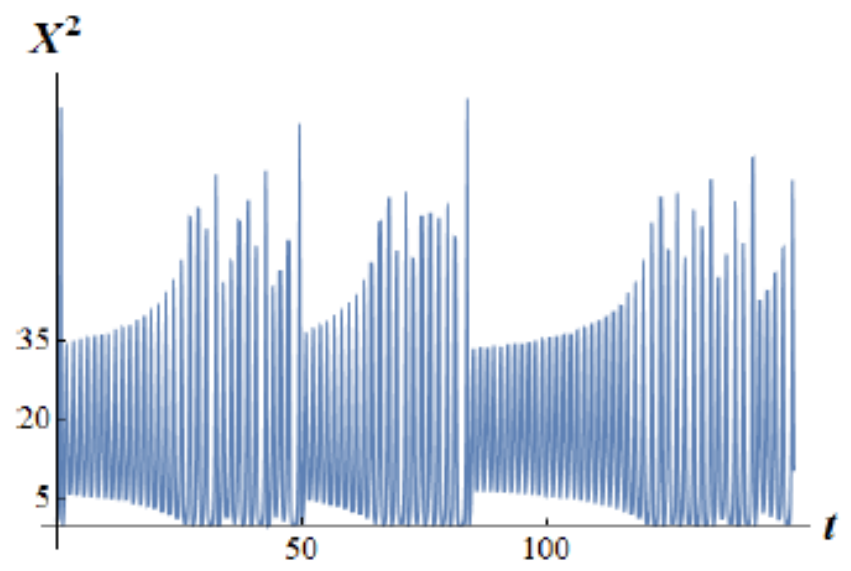

Fig. 4. Evolución temporal de $X^{2}$ para un valor del bombeo $r=19$, inferior al de la bifurcación de Hopf $r_{H B}=21$ para $\sigma=3$; $b=1$. HME con c.i. $\left\{X_{0}=Y_{0}=1 ; Z_{0}=5\right\}$ muy alejadas de la solución estacionaria $\{X=Y=4,2 ; Z=18\}$. Se observa que el valor de la solución obtenida es acotado, pero su evolución no es ni estacionaria ni periódica.

En este caso, implementamos esta HME con un bombeo, $r=19$, inferior a $r_{H B}=21$ pero con unas condiciones iniciales que difieren de la solución estacionaria en más de un 60\%. Los resultados de este cálculo se muestran en la Fig. 4 donde se observa que, para un valor de bombeo correspondiente a la región de estabilidad en el caso de la SME, obtenemos ahora una solución no estacionaria.

Observamos en segundo lugar qué sucede para valores del bombeo superiores al de la bifurcación. Elegimos ahora un bombeo ligeramente superior a la bifurcación, $r=21,5$, pero manteniendo perturbaciones pequeñas con SME. El resultado aparece representado en la Fig. 5 donde se observa, de nuevo, que obtenemos una solución no estacionaria.

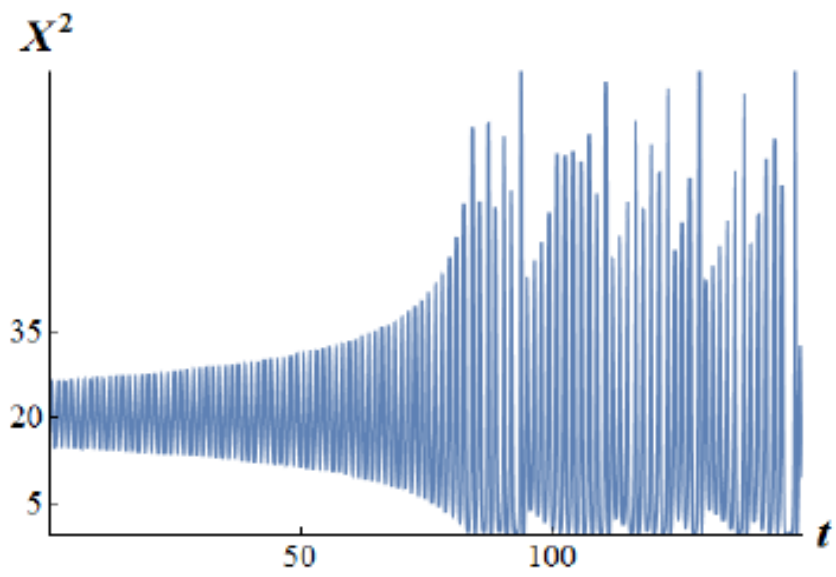

Fig. 5. Evolución temporal de $X^{2}$ para un valor del bombeo $r=21,5$, superior a la bifurcación de Hopf $r_{H B}=21$ para $\sigma=3 ; b=1$. SME con c.i. $\{X=Y=4,2 ; Z=19\}$. Se observa que la solución obtenida es acotada, pero su evolución no es ni estacionaria ni periódica.

El comportamiento de las soluciones dinámicas obtenidas en estos dos ejemplos no es ni estacionario ni periódico, tal y como hemos señalado en las figuras. Este comportamiento dinámico observado por primera vez a partir de la solución de las ecuaciones de Lorenz es el denominado caos determinista.

Estas dos gráficas de evolución temporal nos han permitido observar su perfil característico y

también plantear dos preguntas fundamentales: por una parte ¿cómo es ese comportamiento caótico y por qué se define así? y, por otra, ¿qué conexión existe entre el caos y otros comportamientos más regulares como las oscilaciones periódicas que aparecen para otros valores de $r$ (como el ejemplo de la Fig. 6)? 


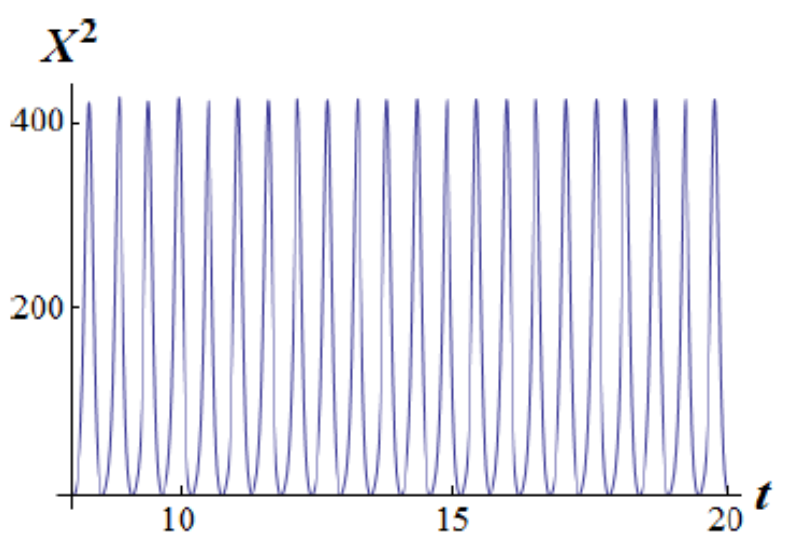

Fig. 6. Evolución temporal de $X^{2}$. Valores de los parámetros $\sigma=3 ; b=1 ; r=150$. La solución dinámica, después de un régimen transitorio breve, muestra un comportamiento periódico.

La primera cuestión la respondemos en el apartado 3 con el estudio de la solución caótica y la segunda la abordaremos en el apartado 4 relativo a las rutas al caos.

\section{Comportamiento caótico}

La existencia de la solución que hemos denominado caótica es sin duda sorprendente. Recalquemos que estamos hablando de una solución de unas ecuaciones diferenciales ordinarias deterministas y recordemos que a este tipo de ecuaciones corresponde una solución única fijados los parámetros y la condición inicial. ¿Cómo es posible que esas ecuaciones proporcionen una solución que no es ni periódica ni cuasiperiódica, y con aspecto tan irregular que uno diría que tiene una componente aleatoria?

Así, en este régimen caótico el estado futuro del sistema es difícilmente predecible, como vamos a comprobar a continuación. Tal y como hemos mencionado en la introducción, la característica definitoria del caos es la sensibilidad a las condiciones iniciales. Significa esto que dos trayectorias que se inician en puntos muy próximos del espacio de fases se separan exponencialmente con el tiempo. Esta propiedad definitoria del régimen caótico hace que las predicciones prácticas en sistemas complejos, donde es imposible conocer la condición inicial exactamente, sean imposibles transcurrido cierto lapso relativamente breve de tiempo; como sucede, por ejemplo, en la predicción del tiempo atmosférico.

De esta forma, vemos que, a pesar de la sorpresa que produjo la formulación del principio de incertidumbre en la mecánica cuántica, la impredictibilidad no es una característica que sólo se encuentre en ese contexto, sino que también aparece en la mecánica clásica, aunque por motivos muy diferentes.

Con la finalidad de clarificar el significado de la sensibilidad a las condiciones iniciales, elegimos, dentro del conjunto de soluciones a las ecuaciones de Lorenz, dos tipos de comportamientos dinámicos diferentes: en un caso el comportamiento será periódico y, en el otro, caótico. El estudio consistirá en elegir condiciones iniciales muy próximas en los dos casos, con una diferencia del orden del $0,01 \%$, y observar cómo influyen en el estado que esperamos encontrar el sistema después de un lapso de tiempo determinado.

Elegimos primero una solución periódica. Por ejemplo, la de la Fig. 6. En ella se puede observar que la solución dinámica, después de un régimen transitorio breve, muestra un comportamiento periódico.

En la Fig. 7, representamos de tres modos distintos la diferencia entre la solución elegida y otra correspondiente a unas condiciones iniciales ligeramente diferentes. Así, en la Fig. 7(a) hemos representado la evolución de esta diferencia y comprobamos que a partir de un periodo transitorio breve los valores alcanzados por las dos soluciones se aproximan significativamente hasta casi igualarse, excepto por unas oscilaciones acotadas y del mismo perfil periódico que la solución encontrada. En la Fig. 7 (b) representamos esta diferencia a mayor escala y superado el periodo transitorio para observar la poca relevancia de la diferencia de las condiciones iniciales con mayor detalle. Finalmente, en la Fig. 7(c) se muestra esta diferencia en escala logarítmica con el objeto de enfatizar la escasa relevancia de la diferencia entre las soluciones. 

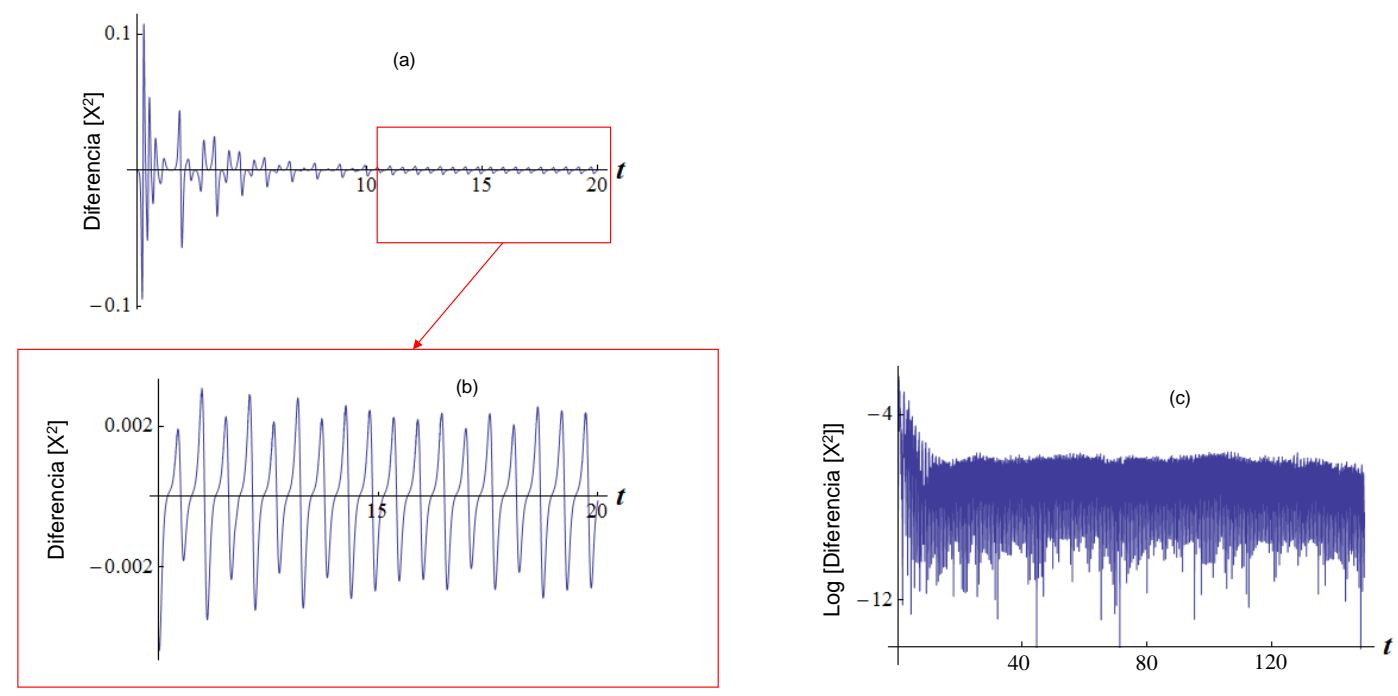

Fig. 7. Comparación de la evolución temporal de $X^{2}$ para dos conjuntos de condiciones iniciales muy próximos: $C I_{1}=\left\{X_{0}=Y_{0}=1\right.$; $\left.Z_{0}=13\right\} ; C I_{2}=\left\{X_{0}=Y_{0}=1+10^{-4} ; Z_{0}=13+10^{-4}\right\}$. En (a) representamos la diferencia entre las dos soluciones. En (b) vemos que la diferencia entre las soluciones casi desparece, excepto por unas oscilaciones acotadas y del mismo perfil periódico que la solución encontrada. En (c) representamos el logaritmo de la diferencia estudiada.

Realizamos ahora el mismo análisis para el comportamiento que denominamos caótico, alcanzado para un valor de bombeo $r=20,8$ inferior al de la bifurcación de Hopf, $\left(r_{H B}=21\right)$ y condiciones iniciales $\left\{X_{0}=Y_{0}=1\right.$; $\left.Z_{0}=4\right\}$-método de Hard-Mode Excitation-

En la Fig. 8 representamos la diferencia entre la solución elegida y otra correspondiente a unas condiciones iniciales ligeramente diferentes. Así, en la Fig. 8(a) hemos representado superpuestas estas dos soluciones para poder observar si la diferencia entre ellas es predecible en su evolución temporal. Se observa en esta representación que a partir de un periodo transitorio breve (Fig. 8(b)) los valores alcanzados por las dos soluciones son sensiblemente diferentes sin seguir aparentemente ningún patrón temporal en esa diferencia. Por último, en la Fig. 8(c) se muestra esta diferencia en escala logarítmica.
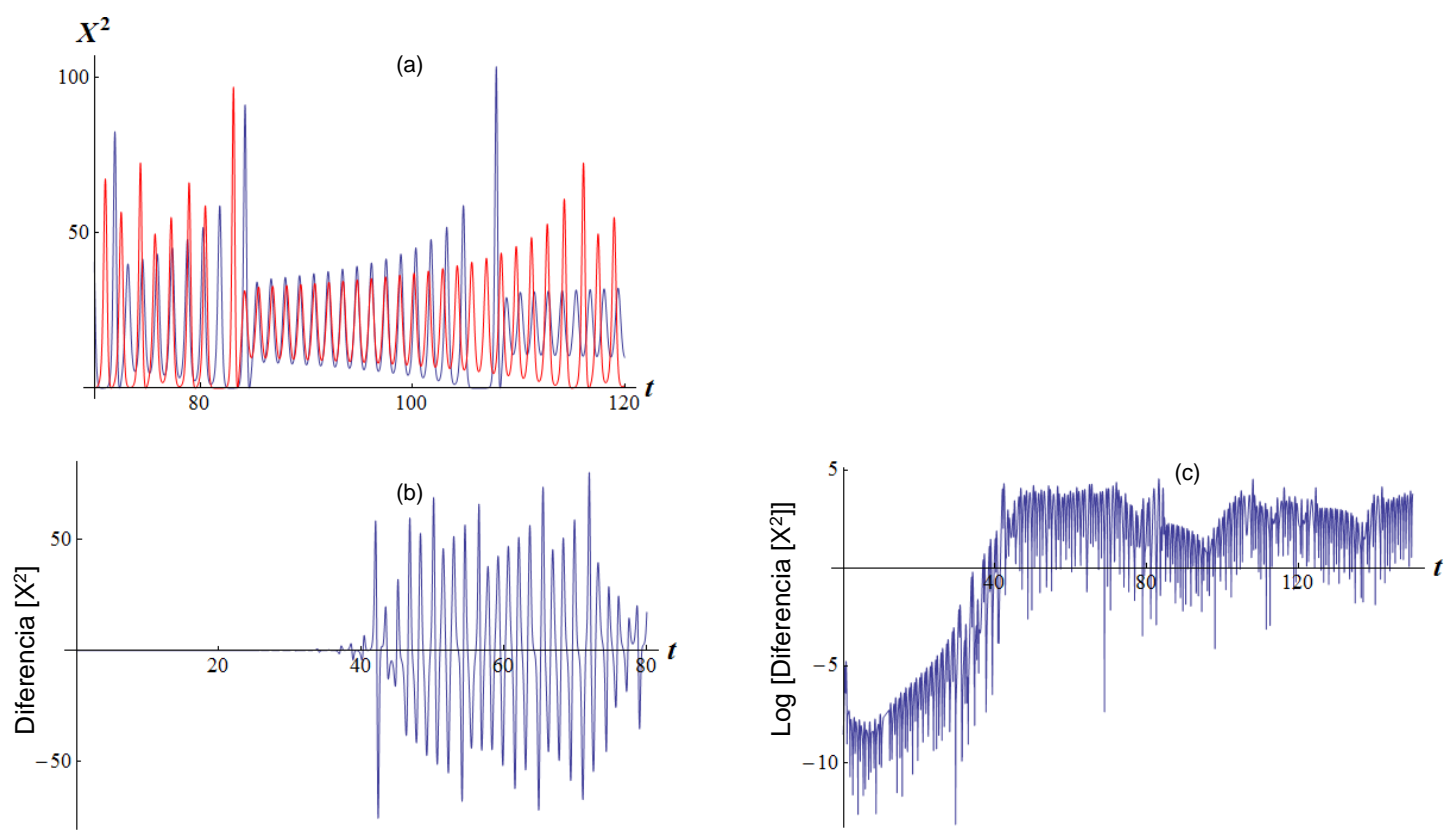

Fig. 8. Comparación de la evolución temporal de $X^{2}$ para dos conjuntos de condiciones iniciales muy próximos: $r=20,8 ; C I_{1}=$ $\left\{X_{0}=Y_{0}=1 ; Z_{0}=4\right\} ; C I_{2}=\left\{X_{0}=Y_{0}=1+10^{-4} ; Z_{0}=4+10^{-4}\right\}$. En (a) observamos la superposición de las dos soluciones. En (b) vemos que la diferencia entre las soluciones, después de un régimen transitorio breve, aumenta drásticamente. En (c) hemos representado la evolución temporal del logaritmo de la diferencia estudiada. 
En resumen, las ecuaciones de Lorenz, como modelo para calcular el estado de un sistema físico, predicen la aparición del denominado caos determinista para ciertos valores de los parámetros que caracterizan el estado del sistema. Este comportamiento caótico se distingue por introducir una alta impredictibilidad en la evolución temporal y esa incertidumbre proviene de la gran sensibilidad a las condiciones iniciales que presentan algunas soluciones a esas ecuaciones. El hecho de que en los experimentos o estudios de sistemas físicos reales el margen de error en la medición de las condiciones iniciales sea en muchas ocasiones superior al que hemos utilizado para comprobar la divergencia arbitraria en las soluciones, hace que el régimen caótico se caracterice, principalmente, por la impredictibilidad de la evolución de estos sistemas.

\section{Mapa de las soluciones dinámicas. Rutas al caos}

La intención de este apartado es trazar un mapa de qué tipo de soluciones podemos encontrar al movernos en el espacio de parámetros. Esta tarea es sumamente compleja ya que aparte de las tres dimensiones del citado espacio $(\sigma, b, r)$ hay que tener en cuenta que, en determinadas regiones, el sistema caerá a uno u otro tipo de soluciones dependiendo de las condiciones iniciales como hemos visto al estudiar los diferentes resultados obtenidos con la Hard Mode Excitation y la Soft Mode Excitation..

Para ilustrar esta idea de mapa en el espacio de parámetros hemos realizado una representación esquemática en la Fig. 9 considerando una sola dimensión. Aparecen tres tipos de soluciones: estacionaria, caótica y periódica; que ya hemos analizado en los apartados anteriores. Pero, además, cada tipo de solución presenta una determinada evolución según el intervalo de bombeo en el que nos encontremos y, en algún caso particular, según las condiciones iniciales escogidas. Esta evolución de cómo cambia el tipo de solución es lo que analizamos en este apartado del trabajo. Analizaremos brevemente en qué consiste la biestabilidad y con mayor detenimiento qué significa el doblamiento de periodos y las intermitencias.

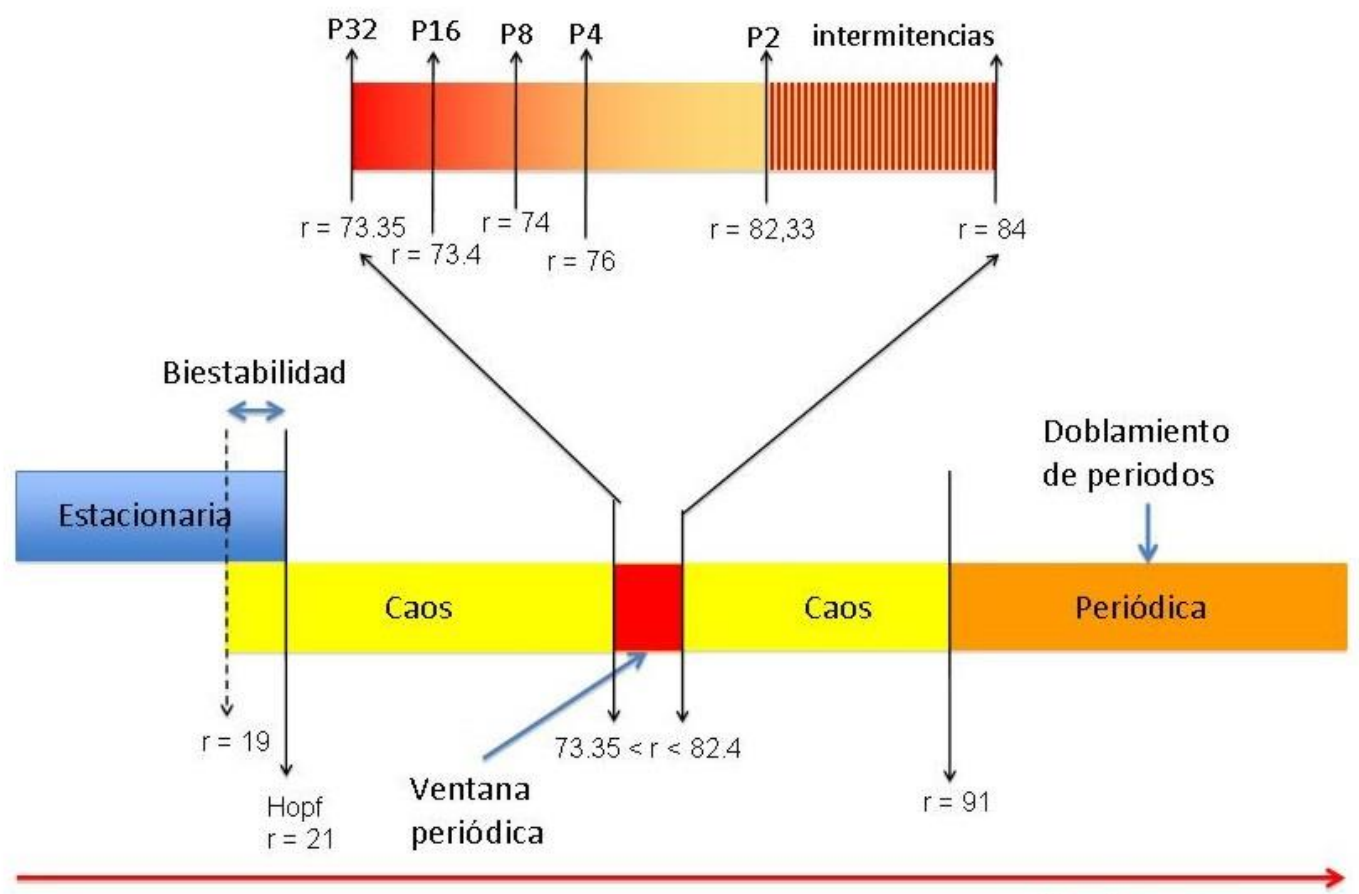

Bombeo : $r$

Fig. 9. Mapa del tipo de soluciones a las ecuaciones de Lorenz-Haken en función del parámetro de control, $r$, con los valores fijos de $\sigma=3$ y $b=1$. La descripción de los diversos tipos de comportamientos se desarrolla con detalle en el texto. Nótese que hay más ventanas periódicas que las marcadas en la figura, en la que sólo se resalta una de ellas. 


\section{4.a. Biestabilidad}

Una de las características novedosas de los sistemas no lineales es que se pueden presentar dos soluciones estables distintas para un conjunto dado de parámetros el sistema. En este caso, que el sistema caiga a una u otra solución dependerá de las condiciones iniciales.

Esta propiedad aparece en el modelo de Lorenz en los alrededores de la bifurcación de Hopf, tal y como ya hemos visto anteriormente al hablar de las técnicas de Hard-Mode y Soft-Mode Excitation (véase la Fig. 4).
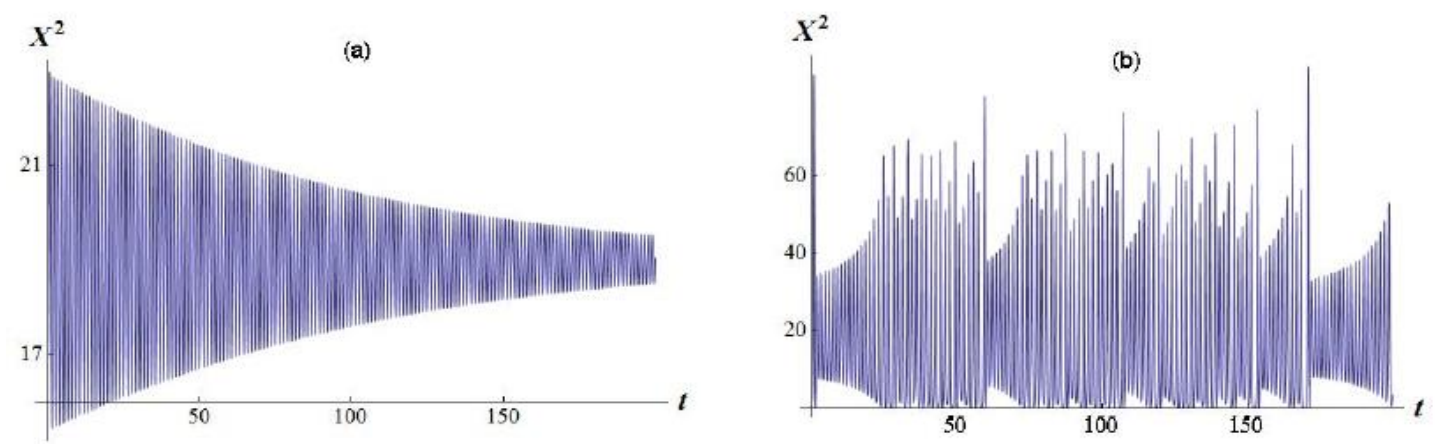

Fig. 10. Evolución temporal de $X^{2}$ para $r=20<r_{H B}=21$. (a) $\operatorname{SME}$ con c.i. $\left\{X_{0}=Y_{0}=4,1 ; Z_{0}=18\right\}$; la solución obtenida decae hacia la solución estacionaria. (b) HME con c.i. $\left\{X_{0}=Y_{0}=1 ; Z_{0}=5\right\}$; la solución obtenida presenta un comportamiento caótico.

Representamos en la Fig. 10 dos resultados diferentes obtenidos para el mismo valor del bombeo con el objetivo de mostrar de forma clara en qué consiste esa biestabilidad.

Observamos, por tanto, que para el valor de $r=20$ las soluciones pueden corresponder a estados diferentes del sistema, dependiendo de las condiciones iniciales. Esta es la propiedad denominada biestabilidad.

\section{4.b. Ruta de doblamiento de periodos}

Al estudiar sistemas que muestran comportamiento caótico se puede observar que, en función de la variación del parámetro de control, este régimen caótico no suele presentarse súbitamente, sino que suele venir precedido por unos comportamientos característicos que se suceden a través de una serie de bifurcaciones desde una solución periódica. Las secuencias que llevan del comportamiento periódico al caótico son denominadas rutas al caos y se han estudiado sistemáticamente, dentro de la variedad de tipificaciones que se han encontrado. Las más usuales [8-10] son la ruta de doblamiento de periodos, o de Feigenbaum, la de cuasiperiodicidad, o de Ruelle-Takens, y las rutas de intermitencias. Estas últimas rutas incluyen los tres tipos de Pommeau y Manneville, la intermitencia on-off y algunas más. El modelo de Lorenz presenta dos de estos comportamientos: la ruta de Feigenbaum y las de intermitencias [2]; en este subapartado nos centramos en la ruta de Feigenbaum y en el siguiente desarrollamos un poco más la de intermitencias.

En el modelo de Lorenz la ruta de Feigenbaum aparece de forma inversa, es decir, para $r>>r_{H B}$ el comportamiento es periódico y a medida que $r$ va disminuyendo, el periodo del atractor se va multiplicando por dos según se va cruzando una serie de bifurcaciones. Cada atractor de periodo más largo tiene un dominio de existencia cada vez menor en términos de $r$, de forma que es difícil encontrar periodos superiores a 8 o 16 veces la duración del período inicial. Tras esta secuencia, aparece el comportamiento caótico.

Uno de los apartados de la práctica planteada consiste en caracterizar la ruta de Feigenbaum en función de $r$. Para caracterizar esta ruta se debe integrar las ecuaciones durante un lapso y analizar el comportamiento observado. En la Fig. 11 se representa la evolución temporal de la solución dinámica para los valores del bombeo $r_{1}=150, r_{2}=120, r_{3}=98, r_{4}=96,8$. En estas gráficas se puede observar la aparición de un período cada vez mayor conforme vamos bajando el valor del bombeo. Finalmente, para $r_{4}=91$ y las mismas condiciones iniciales se puede comprobar que la solución cae en el régimen caótico.

\section{4.c. Ventanas periódicas. Intermitencias}

Si echamos un vistazo más cuidadoso en la zona que hemos marcado como caótica, vemos que existen en su interior dominios (pequeños normalmente) en los que el comportamiento es periódico (Fig. 9). A estos dominios se les denomina ventanas periódicas. Cabe decir, sin embargo, que estas soluciones periódicas son diferentes a las que hemos encontrado antes como se explica a continuación. 
Si la ruta seguida es el aumento del parámetro de control $r$, para entrar en estas ventanas desde el caos se atraviesa un doblamiento inverso de periodos, mientras que para salir y volver al caos se atraviesa una región de intermitencia. Esta intermitencia se caracteriza por la alternancia, en la misma serie temporal, de fases de comportamiento prácticamente periódico (llamadas fases laminares) y súbitos estallidos (o bursts) de comportamiento irregular. Al inicio, para un cierto valor de $r$ aparecen los primeros bursts siendo estos breves y largas las fases laminares. Después, al variar $r$ alejándonos de la bifurcación, la duración de las fases laminares se va acortando hasta que para un cierto valor de r el comportamiento es completamente caótico. Dependiendo de las propiedades estadísticas de la duración de las fases laminares, la intermitencia se clasifica como de tipo I, II o III [2]. No entramos en este texto en la discusión y caracterización de las intermitencias, pero sí queremos explicitar que, en este caso, son del tipo I.
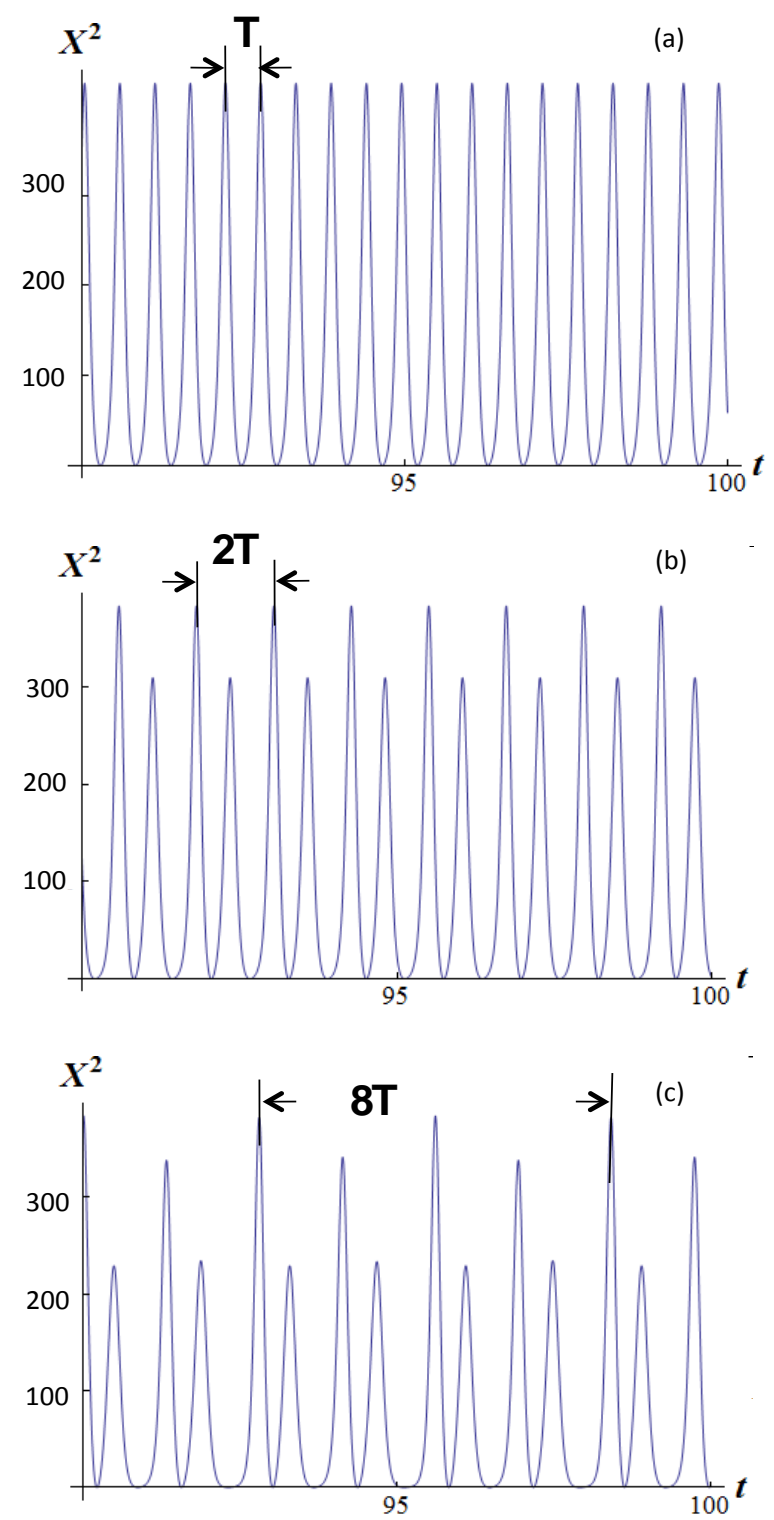

Fig. 11. Evolución temporal de $X^{2}$ para diversos valores de $r$. HME con c.i. $\left\{X_{0}=Y_{0}=2 ; Z_{0}=5\right\}$. (a) $r_{1}=150$; comportamiento periódico. (b) $r_{2}=120$; comportamiento periódico con un periodo de doble duración que el anterior; (c) $r_{4}=96,8$; comportamiento periódico ocho veces más largo que el del primer ejemplo

Aunque para $\sigma=3$ y $b=1$ existen varias ventanas periódicas, para ilustrar este apartado hemos escogido un solo intervalo, $73,35 \leq r \leq 82,40$, en el que se puede observar el comportamiento típico de la ruta de intermitencias. Así, en la Fig. 12 mostramos los cambios sufridos por la solución a partir de $r=82,33$; que presenta un comportamiento netamente laminar o periódico con dos máximos distintos dentro de cada período. Al aumentar el valor del bombeo, $r$, podemos observar que, en un intervalo de tiempo 
determinado, aparece sólo un burst, como en la Fig. 12(a); luego, para un valor de $r$ superior, varias intermitencias, como en la Fig. 12(b) y finalmente, para valores de $r$ superiores, volvemos al régimen caótico (ver la Fig. 12(c)).
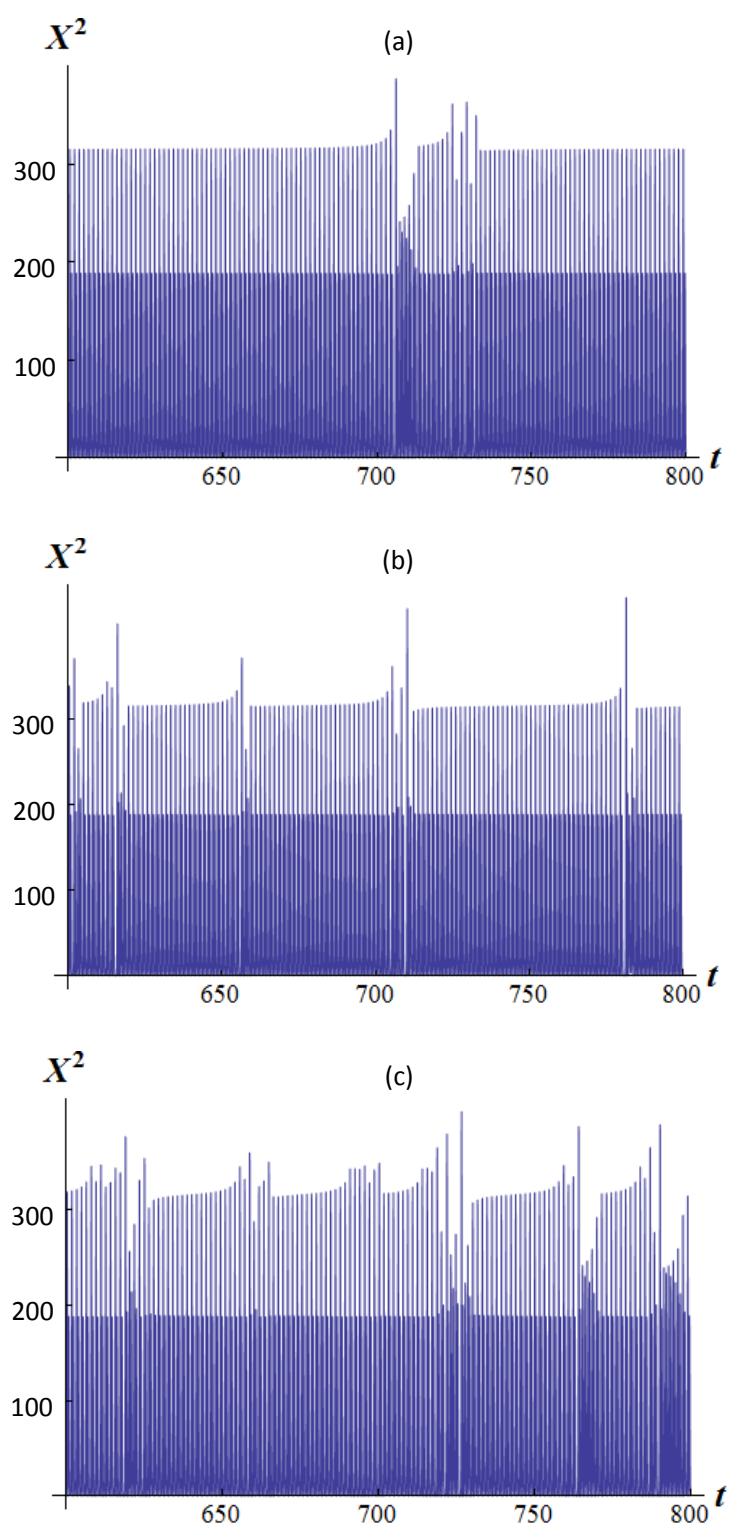

Fig. 12. Evolución temporal de $X^{2}$ para diversos valores de r. Para $r=82,34$ (a) se observa como el comportamiento periódico es interrumpido, brevemente, por un comportamiento irregular. Para $\mathrm{r}=82,35$ (b) vemos como las fases de un comportamiento periódico o laminar alternan con estallidos, irregulares. Las fases laminares son cada vez más breves y en $r=82,4$ (c) el comportamiento ya es completamente caótico.

Dentro del mismo intervalo, si reducimos a partir de $r=82,33$ el valor del bombeo, $r$; aparece de nuevo el doblamiento de periodos que ya vimos en el apartado anterior. En este caso hemos observado el paso a períodos con cuatro máximos en $r=76$, ocho en $r=74$, dieciséis en $r=73,4$; y treinta y dos en $r=73,35$. A partir de ese punto podemos observar de nuevo el perfil caótico, intermitencias y doblamientos de periodo.

En la Fig. 9 mostramos, en la zona de ventana periódica, todo este proceso de entrada en la ventana a través de las intermitencias y salida a través del doblamiento de periodos.

Con el estudio de este apartado hemos analizado un ejemplo de ventana periódica. Podemos preguntarnos por el estudio sistemático de la localización y extensión de estas ventanas periódicas en función la 
variación de $r$; pero esa ampliación exige un análisis mucho más preciso y es impredecible hasta tal punto, que la tarea de realizar un mapa detallado de las diferentes rutas al caos en cada uno de estos supuestos permanece inconclusa.

\section{Otras herramientas de análisis y caracterización del caos}

En este apartado describimos y analizamos brevemente otras herramientas de análisis que pueden ayudar a caracterizar mejor el comportamiento del sistema dinámico, bien sea porque nos permiten distinguir con mayor precisión el tipo de multiplicidad periódica que obtenemos, bien porque pueden ayudar a caracterizar un comportamiento caótico.

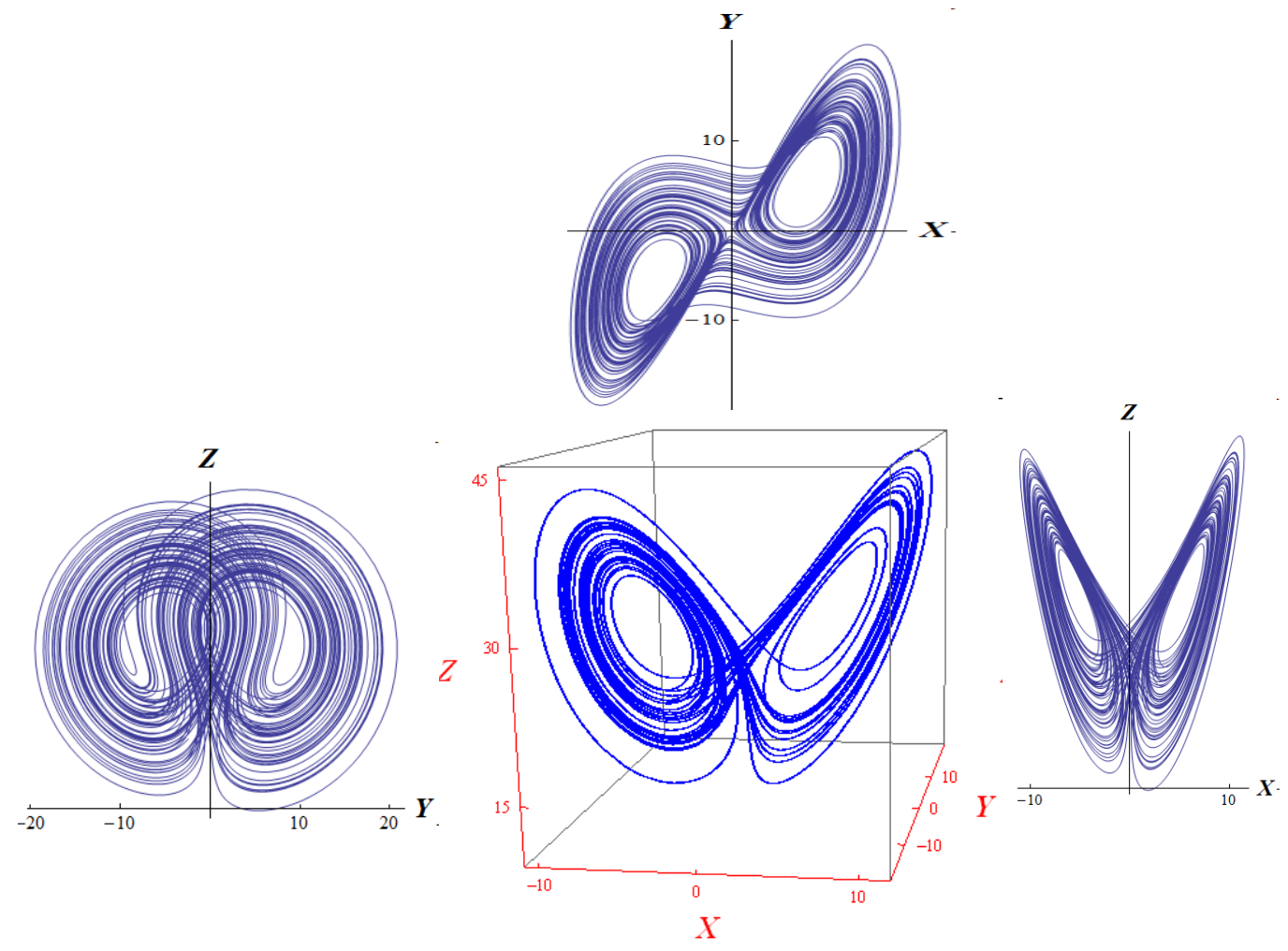

Fig. 13 Representación tridimensional del atractor caótico de Lorenz junto con tres proyecciones bidimensionales para $r=30$

\section{5.a. Representación en el espacio fásico. Atractores}

En el estudio de la dinámica del comportamiento caótico, si se presta atención a la evolución con el tiempo de la trayectoria en el espacio fásico, se ve cómo ésta va recorriendo un conjunto acercándose en algunos momentos mucho a sí misma, para luego volver a alejarse, dibujando una figura característica denominada atractor de Lorenz. Esta descripción se ve en la Fig. 13, en la que se muestran las tres proyecciones bidimensionales y una representación tridimensional del atractor. Así, en este régimen caótico, el estado futuro del sistema es impredecible, tal y como se ha visto en apartados anteriores.

Recordamos que la característica definitoria del caos es la sensibilidad a las condiciones iniciales. Significa esto que dos trayectorias que se inician en puntos próximos del espacio de fases se separan exponencialmente con el tiempo. Para verlo con más detalle, utilizamos los datos de la Fig. 8(a) donde se muestra la diferencia del valor que alcanza $X^{2}$ para dos valores de las condiciones iniciales muy próximas (con una diferencia inferior al $0,01 \%$ ). Se observa claramente que, tras un breve régimen transitorio, los valores divergen arbitrariamente. En la Fig. 14 se observa también la representación tridimensional en el espacio de fases de estas dos trayectorias muy próximas inicialmente y cómo estas trayectorias se separan arbitrariamente después de un cierto intervalo de tiempo. 


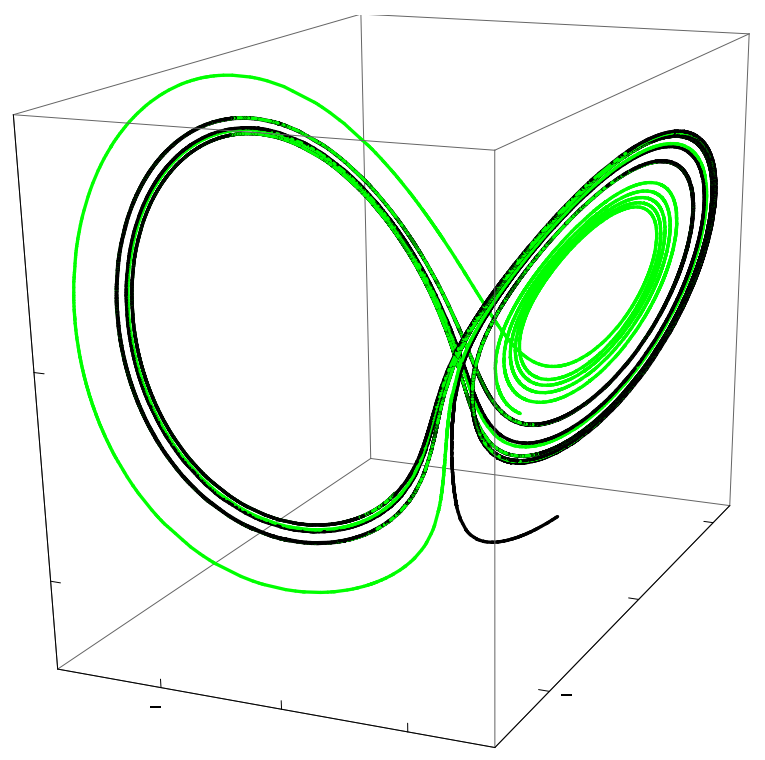

Fig. 14. Diferencia entre las trayectorias en el diagrama de fases tridimensional para la evolución del sistema a partir de dos conjuntos de valores iniciales muy próximos $\left\{X_{1}=Y_{1}=1 ; Z_{1}=4\right\}$ (línea verde) y $\left\{X_{2}=Y_{2}=1+10^{-4} ; Z_{2}=4+10^{-4}\right\}$ (línea negra). Con un valor del bombeo $r=20,8$, cercano a la bifurcación de Hopf $r_{H B}=21$. En este diagrama se representa la evolución del sistema entre $t_{2}=30 u$.t. y $t_{3}=50 u$. $t$. Las trayectorias, una vez superado un periodo inicial breve, se separan arbitrariamente.
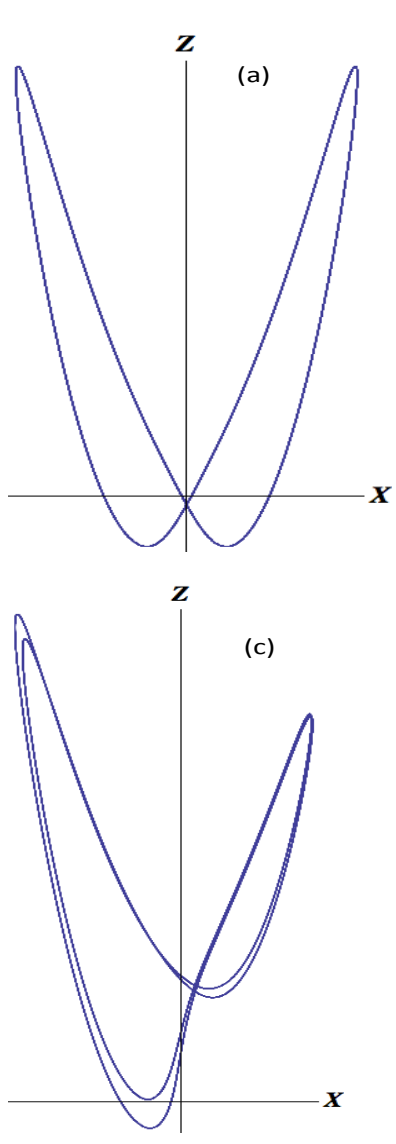
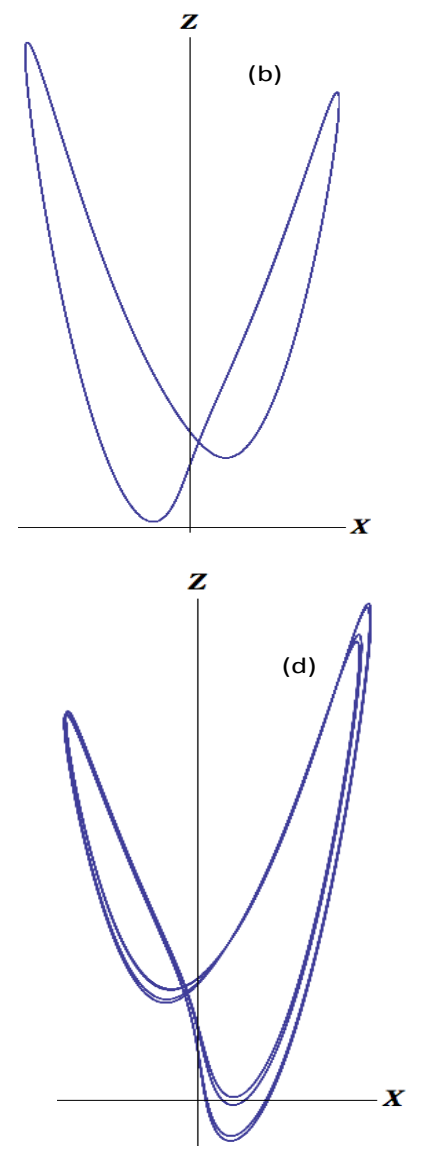

Fig. 15. Proyección del atractor en el plano $(X, Z)$. Condiciones iniciales $\left[X_{0}=Y_{0}=7 ; Z_{0}=90\right]$. (a) Para $r=140$ la trayectoria recorre una línea cerrada o lóbulo simétrico respecto del eje $O Z$; período $T$. (b) Para $r=120$ se observa un lóbulo asimétrico respecto del eje $O Z$; período $2 T$. (c) Para $r=98$ la trayectoria recorre dos lóbulos asimétricos; período $4 T$. (d) Para $r=96,6$ la trayectoria recorre cuatro lóbulos asimétricos; período $8 T$. 
Esta representación en el espacio de fases también es muy útil para caracterizar el comportamiento periódico. Conviene recordar, para poder distinguir los distintos tipos que presenta este comportamiento, que en el caso de un comportamiento periódico sencillo o fundamental la trayectoria dibujada en el espacio de fases es una línea cerrada, o lóbulo, simétrica (cf. la Fig. 15(a)). De acuerdo con lo estudiado en los apartados anteriores, podemos recorrer el camino de doblamiento de periodos a medida que vamos reduciendo el valor del bombeo desde $r=140$ hasta $r=96,6$ para observar las distintas transformaciones que sufre la trayectoria en estos doblamientos. Así, observamos que la aparición de una asimetría implica que se ha doblado el período en la variable $X(t)$ (cf. la Fig. 15(b)) y que cada nuevo lóbulo asimétrico indica la duración del periodo se ha multiplicado por dos, como se observa en la Fig. 15(c) y la Fig. 15(d) en las que se representa el comportamiento periódico con períodos 4 y 8 veces más largos, respectivamente, que el observado en la Fig. 15(a).

\section{5.b. Espectro de Fourier}
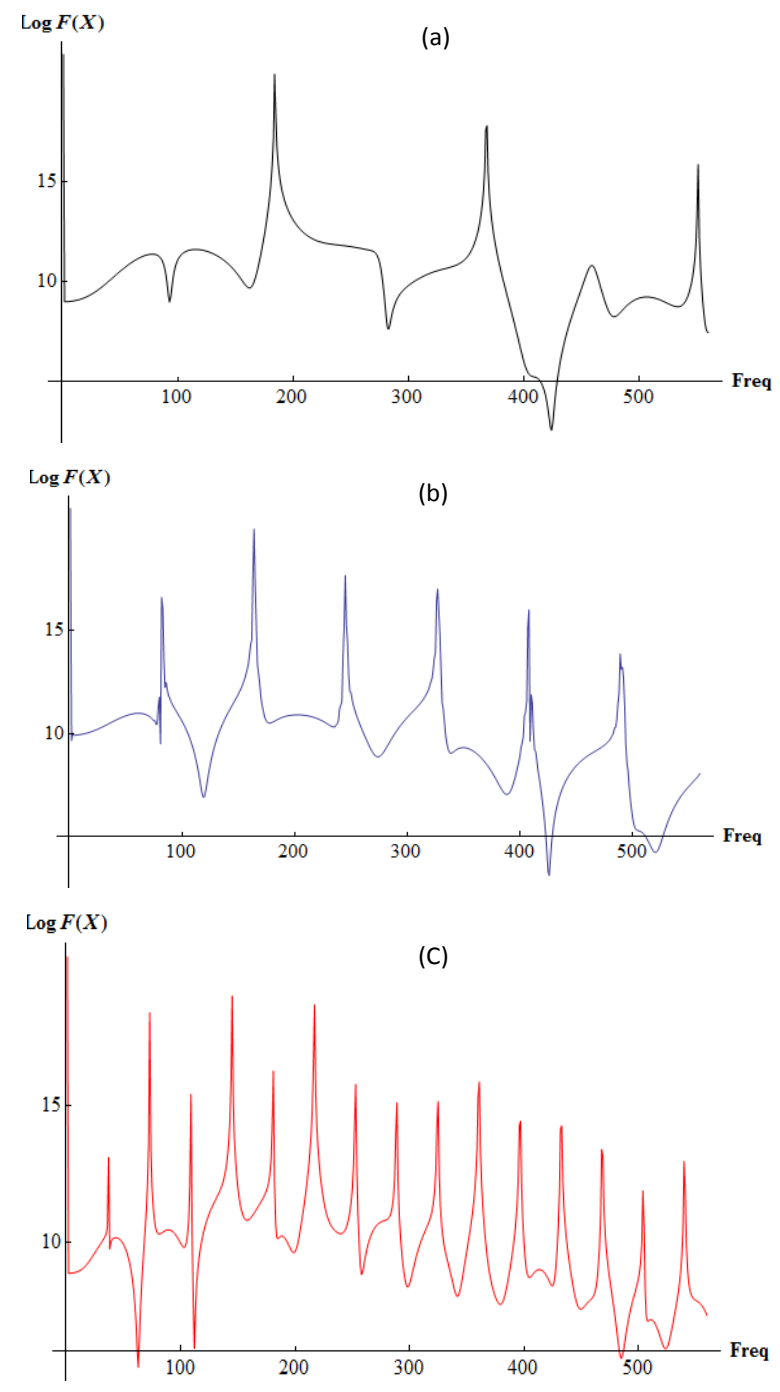

Fig. 16. Logaritmo de la Transformada de Fourier de la variable $X$, entre $t_{1}=0$ u.t. y $t_{2}=100$ u.t., para distintos valores de la inyección $r$ con condiciones iniciales $\left[X_{0}=Y_{0}=0.1 ; Z_{0}=r\right.$-1]. (a) $r=150$; período $T$. (b) $r=120$; período $2 T$. (c) $r=98$; período $4 T$.

Se trata de otra herramienta que nos permite distinguir entre un comportamiento periódico y uno caótico. El comportamiento caótico se caracteriza porque su espectro consta de una banda muy amplia de frecuencias, independientemente de que haya o no picos pronunciados. Como ejemplos de esta característica, en la Fig. 16(a) representamos la transformada de Fourier de la variable $X(t)$ correspondiente a la solución periódica de período T; en la Fig. 16(b) y la Fig. 16(c) representamos la transformada de Fourier de la variable $X(t)$ correspondiente a las soluciones periódicas de período $2 T$ y 
4T, respectivamente. Por último, en la Fig. 17 representamos la misma gráfica para el caso de un comportamiento caótico.

En la Fig. 16, correspondiente al comportamiento periódico, se observa, además, la utilidad de la representación mediante la transformada de Fourier para distinguir las frecuencias fundamentales que existen en la solución periódica, en el fenómeno que denominamos doblamiento de periodos. En este ejemplo comprobamos que el número de picos existentes antes de la frecuencia fundamental nos indica el número de veces que se ha doblado el período.

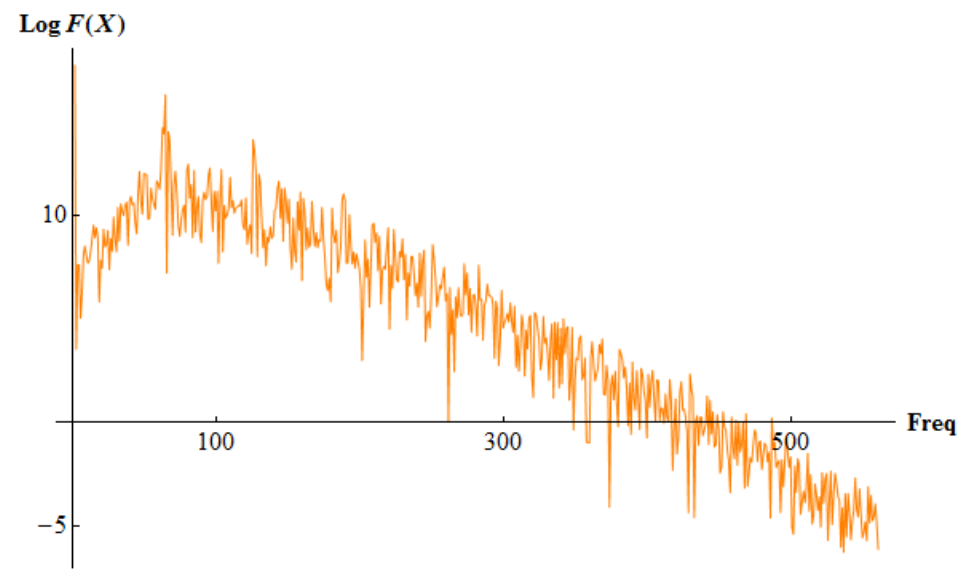

Fig. 17 Logaritmo de la Transformada de Fourier de la variable $X$, entre $t_{1}=0$ u.t. y $t_{2}=100 u$.t., para $r=20,8$ con condiciones iniciales $\left[X_{0}=Y_{0}=0.1 ; Z_{0}=r-1\right]$. Se observa el perfil característico de un comportamiento caótico, con una amplia banda de frecuencias, contrariamente a la Fig. 16..

\section{5.c. Mapa de máximos}

Las representaciones obtenidas hasta ahora nos permiten valorar diversos aspectos del comportamiento del sistema estudiado, tales como la duración del régimen transitorio, el comportamiento periódico, el comportamiento caótico o la existencia de atractores, entre otros. Presentamos ahora una nueva representación, el mapa de máximos, que nos permite obtener, con mayor claridad que las representaciones anteriores, información relativa al número de máximos de valor diferente alcanzados en la evolución temporal de la solución; o, lo que es lo mismo, si se trata de una solución periódica en la que se ha producido doblamiento de períodos o si se trata de un comportamiento caótico.

El mapa de máximos se elabora relacionando cada máximo alcanzado por la variable, en nuestro caso $X^{2}$, con el máximo anterior.
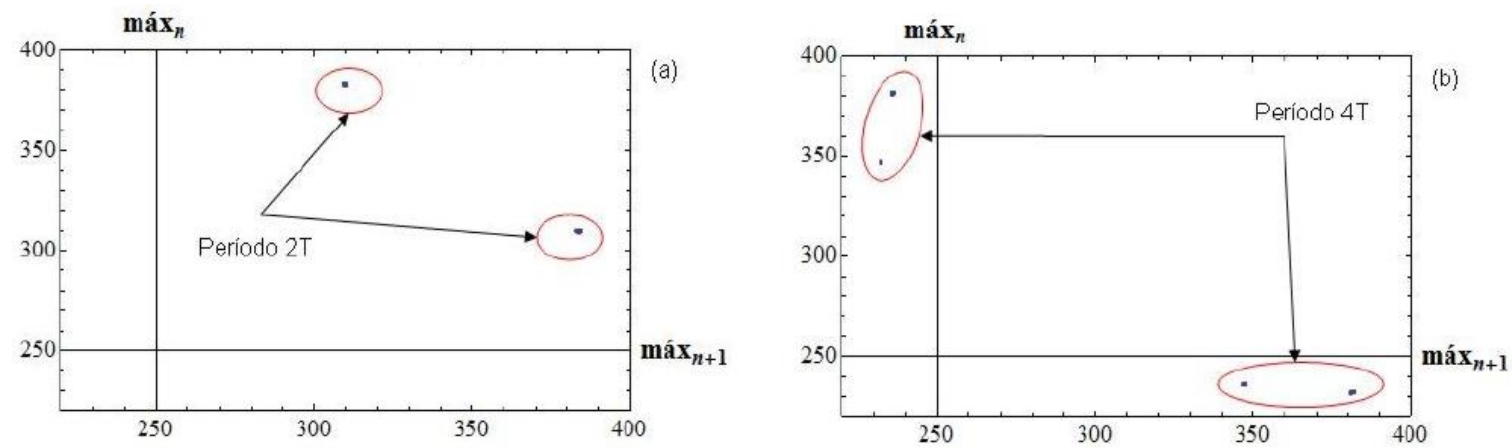

Fig. 18 Mapa de máximos de la variable $X^{2}$ para dos valores del bombeo: $r=120$ (a) y $r=98(b)$. En (a) aparecen dos máximos diferenciados ya que se trata de un comportamiento periódico $P 2$. En (b) aparecen cuatro máximos diferenciados, luego se trata de un comportamiento periódico $P 4$.

Se representan gráficamente el valor del máximo enésimo en el eje de abscisas y el valor del máximo anterior en el eje de ordenadas. Con este método, si los máximos se repiten en el mismo orden, cada uno de ellos aparece como un único punto en el plano formado por los ejes de los valores del máx $x_{n+1}$ y el $m a ́ x_{n}$. Si la solución que estamos estudiando es periódica, el mapa de intensidades es un conjunto finito de puntos, como puede verse en la Fig. 18(a) y la Fig. 18(b) correspondientes a comportamientos de periodos doble y cuádruple respectivamente; mientras que la correspondiente a la solución caótica es el conjunto 
representado en la Fig. 19 en la que se muestra la característica cúspide correspondiente al atractor de Lorenz.

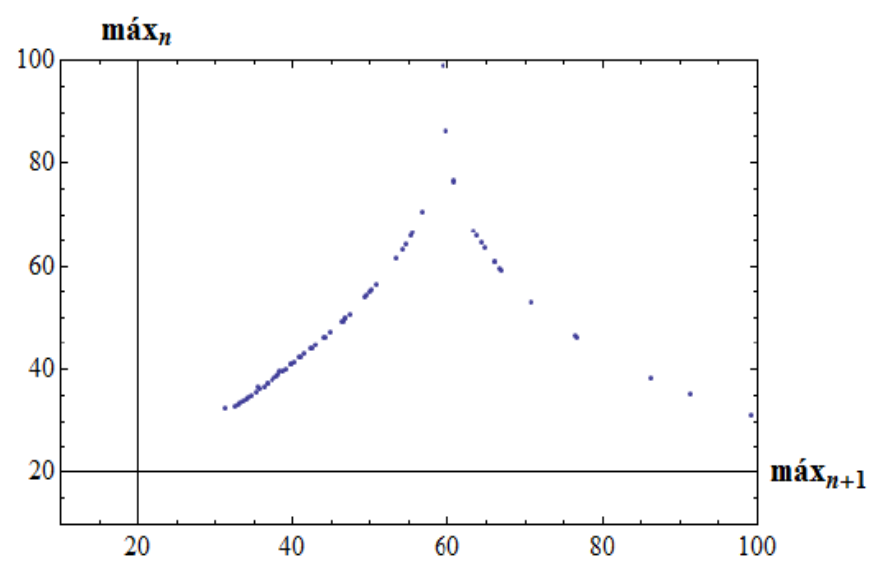

Fig. 19 Mapa de máximos de la variable $X^{2}$ para $r=21$ y condiciones iniciales $\left[X_{0}=Y_{0}=2 ; Z_{0}=5\right]$.

\section{Apuntes para ampliar el estudio del comportamiento dinámico del modelo de Lorenz}

El estudio presentado del comportamiento dinámico del modelo de Lorenz no es, ni pretende ser, exhaustivo. Es más, dado su carácter de iniciación al tema que pretende estudiar, tanto en el análisis teórico general como en la elección de los ejemplos desarrollados, hay algunos aspectos que se pueden profundizar - bien para reflexionar con más detalle sobre las propiedades observadas o bien para conocer mejor la riqueza del modelo - y que en la práctica propuesta se dejan de lado, con la finalidad de que el alumnado llegue con claridad a un conocimiento básico sobre el modelo de Lorenz, la dinámica no lineal y los métodos de cálculo empleados.

En este apartado destacamos algunos de esos aspectos y, basados en la experiencia de muchos otros trabajos realizados en este campo, apuntamos brevemente en qué dirección sería interesante desarrollarlos para ampliar la base que se adquiere con esta práctica.

\section{6.a. Bifurcación subcrítica: biestabilidad antes de la bifurcación de Hopf}

El detalle sobre el que queremos reflexionar al abordar este tema es el hecho de que, para los valores de los parámetros elegidos, el comportamiento que hemos visto aquí para valores de la inyección, $r$, cercanos a la bifurcación de Hopf no es el comportamiento dinámico predicho por el análisis de estabilidad lineal para el modelo de Lorenz. Concretamente, para $\sigma=3$ en la bifurcación de Hopf se pasa bruscamente de un comportamiento estacionario a un comportamiento caótico que nada tiene que ver con las oscilaciones de frecuencia $\Omega_{H B}$ que predice la ecuación (12).

El origen de este resultado radica en que la bifurcación de Hopf del modelo de Lorenz es subcrítica y no supercrítica. En una bifurcación supercrítica, la solución estacionaria pierde su estabilidad en favor de una solución periódica estable cuya amplitud crece monótonamente desde cero al cruzar la bifurcación. En una bifurcación subcrítica, por el contrario, la solución periódica que coalesce con la solución estacionaria en la bifurcación de Hopf es inestable, con lo que no puede ser observada. Así, en una bifurcación subcrítica, el sistema no puede pasar suavemente de la solución estacionaria a unas oscilaciones de pequeña amplitud y lo que ocurre es que pasa de la solución estacionaria (ya inestable) a otra solución no conectada localmente con ella, es decir pasa a cualquier otra solución dinámica que sí sea estable. En nuestro caso, como vimos anteriormente, esa solución es precisamente el atractor caótico extraño que ya existe para valores de $r$ inferiores a los de la bifurcación.

\section{6.b. Otras soluciones dinámicas}

Las soluciones de las ecuaciones de Lorenz que hemos descrito hasta ahora (soluciones estacionarias y soluciones dinámicas, incluyendo caóticas y periódicas) no agotan las soluciones del modelo, existiendo otras soluciones dinámicas diferentes a las que aún no nos hemos referido. 
Un ejemplo es el atractor descrito por C.-Z. Ning y H. Haken [11], que es diferente al que hemos visto hasta ahora (ver la Fig. 20); ya que existe para valores del bombeo muy inferiores a los de la bifurcación de Hopf. Lo interesante de esta solución es que no puede alcanzarse (y por tanto observarse) a no ser que se siga una forma de excitación muy específica.

Para encontrar el atractor de la Fig. 20 se debe hacer una variación de la técnica de hard-mode excitation en la que se parte de condiciones iniciales de láser encendido, en lugar de las de láser apagado, no muy lejos del umbral de encendido (en este caso, por ejemplo, se toman condiciones iniciales correspondientes a $r=5$ ). Esta es la principal razón por la que mencionamos estas soluciones poco conocidas del modelo de Lorenz, porque muestran que la riqueza de soluciones del sistema puede ser muy difícil de describir completamente, debido a que hay soluciones (estables y observables) que sólo pueden excitarse encontrando "la manera adecuada" y ésta puede resultar un tanto exótica.

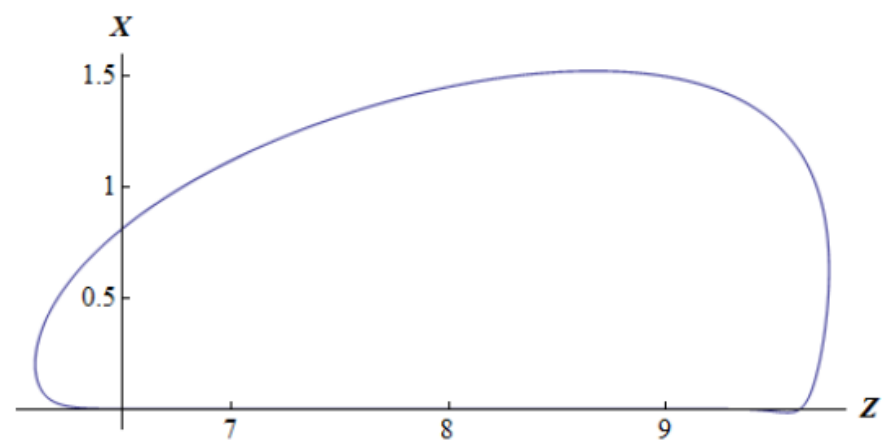

Fig. 20 Proyección en el plano $(X, Z)$ del atractor para $\sigma=2 ; b=0,01 ; r=9,03$ y condiciones iniciales cercanas a la bifurcación de encendido $\left[X_{0}=Y_{0}=0.2 ; Z_{0}=4\right]$. Bombeo inferior a la bifurcación de Hopf: $r_{H B}=10,12121$

En la práctica realizada con los alumnos se puede iniciar este tema con la propuesta de realizar los cálculos con los parámetros escogidos por Ning y Haken $(\sigma=2 ; b=0,01)$. El valor que aparece en este ejemplo es ligeramente inferior al de la bifurcación de Hopf para esos parámetros, con $r_{H B}=10,12121 \mathrm{y}$ $r_{1}=9,03$.

Es posible también realizar un muestreo del tipo de atractor que aparece en función del bombeo, empezando por $r_{1}$ (atractor $O S$ o one-sided), como en la Fig. 20, y descendiendo adiabáticamente hasta ver aparecer la solución que se mueve alrededor del atractor de dos lóbulos (TS o two-sided), como en la Fig. 21.

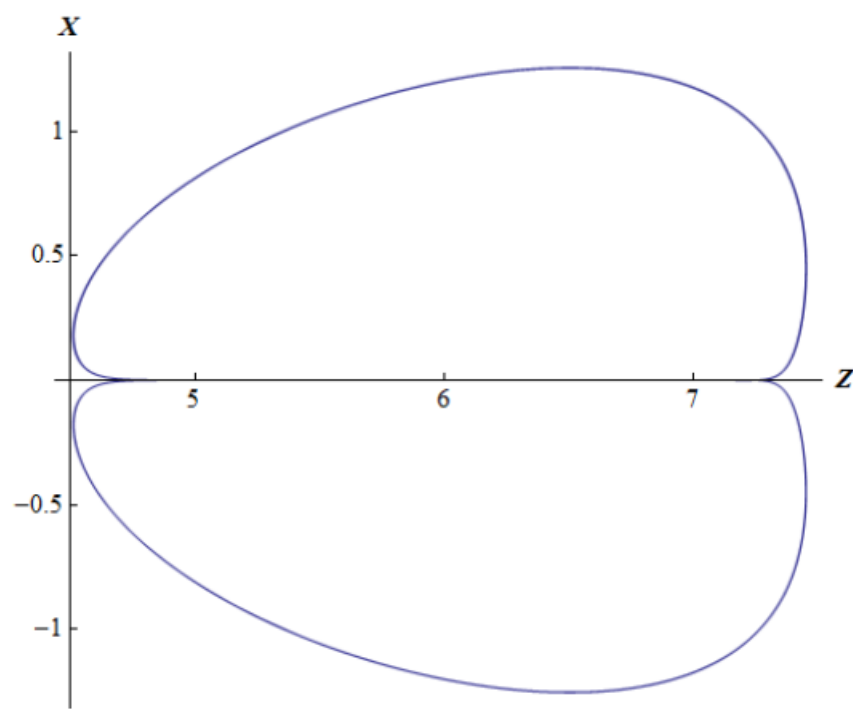

Fig. 21 Proyección en el plano $(X, Z)$ del atractor para $\sigma=2 ; b=0,01 ; r=7,06$ y condiciones iniciales fijadas a partir del descenso adiabático desde la solución de la Fig. 20. Se observa que este atractor periódico es de doble lóbulo o TS.

Debido a la extrema sensibilidad a las condiciones iniciales escogidas, encontrar estos atractores y distinguir la zona de $O S$ o $T S$ requiere de un trabajo muy extenso si lo queremos aplicar a los parámetros escogidos en nuestra práctica. Por este motivo dejamos el tema como una posible ampliación ya sea bien 
de investigación bibliográfica para conocer con más detalles los estudios de Ning y Haken y su posible extrapolación a distintos valores de los parámetros o bien de muestreo del mapa de bifurcaciones del sistema ya estudiado.

\section{6.c. Versatilidad del modelo de Lorenz}

Un aspecto del modelo de Lorenz que tampoco estudiamos en extensión en esta práctica es la gran variedad de ámbitos en los que el modelo se puede aplicar y, por tanto, la diversidad de comprobaciones experimentales de los efectos que predice a las cuales ha dado lugar.

Ya hemos dicho que originalmente el modelo pretendía describir la dinámica atmosférica y que Haken extendió su aplicación al caso de cierto tipo de láseres; sin embargo, hay más: diversos modelos de sistemas mecánicos [12-15], hidrodinámicos [16-18] o incluso económicos [19-21] son isomorfos a las ecuaciones de Lorenz. También son numerosas las observaciones experimentales, como las de Weiss en láseres [22, 23] u otras como las de Illing en norias de agua [24].

Nos podemos preguntar dónde radica esa versatilidad que permite aplicar el modelo en tantos ámbitos. La respuesta se encuentra en la estructura de las ecuaciones (1a)-(1c) y para verlo más claramente introducimos el siguiente cambio de variables $(u, v, w)=(x, y, r-z)$ cuyas ecuaciones se pueden escribir como

$$
\begin{gathered}
\dot{u}=\sigma(v-u), \\
\left(\begin{array}{c}
\dot{v} \\
\dot{w}
\end{array}\right)=\left(\begin{array}{cc}
-1 & u \\
u & -b
\end{array}\right)\left(\begin{array}{l}
v \\
w
\end{array}\right)+\left(\begin{array}{l}
0 \\
R
\end{array}\right),
\end{gathered}
$$

con $R=b \cdot r$. Esta forma de escribir las ecuaciones enfatiza que están formadas por dos bloques: por una parte $(v, w)$ son dos variables disipativas, una de ellas excitada desde el exterior (a través de $R$ ), que están acopladas entre sí de forma cruzada, siendo el parámetro de acoplamiento, $u$, una variable a su vez.

Los sistemas de dos variables como $(v, w)$ son fáciles de encontrar o de diseñar y, teniendo esto, se obtiene la estructura del modelo de Lorenz si se consigue convertir el parámetro de acoplamiento, $u$, en una variable que dependa linealmente de $v$.

Por ejemplo, en el caso del láser $(v, w)$ se relacionan con las variables atómicas, que vienen regidas por esas ecuaciones de forma natural cuando se hace interaccionar el átomo con luz. El paso siguiente es hacer que esa luz sea la generada por los propios átomos y cerrar un circuito de retroalimentación (con los espejos de la cavidad láser) lo que lleva a la ecuación lineal necesaria para $u$.

Entendemos, en resumen, desde este punto de vista que al estudiar la dinámica de sistemas no sea extraño encontrar una estructura de acoplamientos tan sencilla y que, en cualquier caso, también es relativamente fácil de diseñar.

\section{Conclusiones}

En este trabajo hemos expuesto un estudio del modelo de Lorenz tal y como se desarrolla en una práctica de ordenador destinada a estudiantes de un curso de Óptica Cuántica en el último año del grado en Física. Cabe decir, además, que el estudio está diseñado para que sea aplicable en el contexto de otros cursos de dinámica atmosférica, mecánica, oscilaciones, circuitos electrónicos, u otros, ya que nuestro objetivo es también ayudar a introducir contenidos sobre dinámica no lineal en el marco de los grados en ciencias.

Como se ha dicho, esta práctica se desarrolla a lo largo de dos sesiones de tres horas cada una y los estudiantes deben presentar una memoria de su trabajo para ser evaluados. La experiencia ha sido muy bien acogida por parte de los estudiantes y el principal objetivo de este artículo es ponerla a disposición de otras personas con intereses didácticos semejantes a los nuestros. Destacamos, por último, que, para ayudar en ese propósito, los autores proporcionaremos copia del archivo Mathematica utilizado a quienquiera que nos lo solicite.

\section{Agradecimientos}

Este trabajo ha sido llevado a cabo dentro de los proyectos del Ministerio de Educación e Innovación FIS2011-26960 y FIS2014-60715-P 